\title{
Wer spielt Lotto?
}

\section{Umverteilungswirkungen und sozialstrukturelle Inzidenz staatlicher Lotteriemärkte*}

\author{
Jens Beckert • Mark Lutter
}

Zusammenfassung: Auf Basis bevölkerungsrepräsentativer Daten untersuchen wir sozialstrukturelle Umverteilungseffekte des staatlichen Lotteriespiels in Deutschland. Lotterien sind hoch besteuerte wirtschaftliche Transaktionen, deren Einnahmen zugleich einen signifikanten Anteil am öffentlichen Haushaltsbudget darstellen. Unsere Analyse zeigt, dass Lotterien eine Form der regressiven Besteuerung darstellen. Ferner zeigen wir am Beispiel der Breitensportförderung, dass die hierfür zweckgebundene Verwendung staatlicher Lotterieeinnahmen die Regressivität der Besteuerung nicht verringert, sondern noch verstärkt. Innerhalb zentraler soziodemografischer Kategorien wie Einkommens-, Bildungs-, Alters- und Migrationsschichten können wir steuerliche Umverteilungswirkungen nachweisen. Von diesen Befunden ausgehend, diskutieren wir im Schlussteil Implikationen zur Verwendung dieser Einnahmen.

Stichworte: Lotterien · Glücksspiel · Steuern · Umverteilung

\footnotetext{
Jens Beckert $(\bowtie)$

Max-Planck-Institut für Gesellschaftsforschung (MPIfG), Paulstr. 3,

50676 Köln, Deutschland

E-Mail: Beckert@mpifg.de

Mark Lutter

Max-Planck-Institut für Gesellschaftsforschung (MPIfG), Paulstr. 3,

50676 Köln, Deutschland

E-Mail: Lutter@mpifg.de
}

Abstract: On the basis of representative survey data, we examine the distribution effects state lotteries have on the social structure of Germany. Lotteries are highly taxed economic transactions whose proceeds make up a significant share of public fiscal revenues. Our analysis shows that lotteries are a form of regressive taxation. Further,

* Wir danken Fatih Dilekci für seine Hilfe bei der Datenbeschaffung im zweiten Teil der empirischen Analyse. Für hilfreiche Kommentare danken wir Christian Bergs, Steffen Ganghoff, Roberto Garvía, Lothar Krempel und Jörg Rössel. Die Forschungen für diesen Artikel wurden von der Deutschen Forschungsgemeinschaft im Rahmen des Projektes „Nachfrageverhalten und Verteilungswirkungen des Lotteriespiels in Deutschland“" gefördert. 
taking the funding of amateur sports as an example, we show that earmarking lottery money increases the regressivity of this tax rather than reducing it. Using key demographic indicators such as levels of income, education, age and migration, we demonstrate the effects of fiscal redistribution. Based on these findings, we discuss implications of earmarking lottery revenues.

Keywords: Lotteries $\cdot$ Gambling $\cdot$ Taxes $\cdot$ Redistribution

\section{Einleitung}

Lotterien sind Instrumente der Umverteilung. Dies gilt in dreifacher Hinsicht: Zum einen erklären sich die Spieler damit einverstanden, dass ihr Spieleinsatz so umverteilt wird, dass eine nach dem Zufallsprinzip ermittelte kleine Gruppe der Spieler, die Gewinner, den gesamten zur Ausschüttung kommenden Betrag für sich vereinnahmen. Die große Mehrheit der Spieler verliert hingegen ihren Spieleinsatz.

Zum zweiten sind Lotterien insofern Instrumente der Umverteilung, als nicht der gesamte Spielumsatz, abzüglich der Kosten für die Durchführung der Lotterie, an die Gewinner ausgeschüttet wird, sondern nur ein Teil. Die Umverteilung findet in diesem Fall nicht zwischen den Spielern statt, sondern zwischen Spielern und dem Staat. Im deutschen Lotto werden ungefähr 48 Prozent der Spieleinsätze an die Gewinner ausgeschüttet. Mit 13 Prozent der Einsätze werden die Kosten der Durchführung der Lotterie gedeckt, 39 Prozent gehen als Steuern bzw. als Konzessionsabgaben an den Staat. Lotterien sind damit eine besonders hoch besteuerte wirtschaftliche Transaktion. Historisch betrachtet waren Lotterien wesentlich ein Instrument der Finanzierung öffentlicher Ausgaben, womit die Absicht der Einnahmeerzielung im Vordergrund der staatlichen Organisation von Lotterien steht. So diente z. B. die erste in Deutschland durchgeführte Lotterie, die 1611 in Hamburg stattfand, der Finanzierung eines Werk- und Zuchthauses (Paul 1978: 38). Derzeit werden in Deutschland jährlich rund fünf Mrd. Euro aus staatlich konzessionierten Glücksspielen vom Staat vereinnahmt, das Lottospiel allein steuert mit etwa zwei Mrd. Euro den größten Anteil dazu bei.

Schließlich sind Lotterien, möglicherweise zumindest, Instrumente der Umverteilung in einer dritten Hinsicht. Sollten nicht alle Bevölkerungsschichten sich gleichermaßen an dem Lotteriespiel beteiligen und die mit den Lotterieeinnahmen staatlich finanzierten Güter nicht von allen Bevölkerungsschichten gleichermaßen nachgefragt werden, wären Lotterien auch ein Instrument staatlicher sozialer Umverteilung. Die vom Staat durch Lotterien vereinnahmten Mittel lassen sich dabei unter denselben normativen Kriterien von Steuergerechtigkeit beurteilen, wie sie für andere Einnahmequellen der öffentlichen Haushalte angelegt werden. Daraus ergeben sich zwei Fragen: Welche Bevölkerungsgruppen tragen durch überdurchschnittliche Spielbeteiligung überproportional hohe Anteile zu dem Einnahmenaufkommen der Länder aus dem Lottospiel bei? Welche Bevölkerungsgruppen profitieren insbesondere von den durch die staatliche Lotterie finanzierten Gütern?

In diesem Artikel gehen wir diesen beiden Fragen der sozialstrukturellen Verteilungswirkung des Lotteriespiels in Deutschland nach. Die Problematik sozialer Umverteilung 
durch Lotterien gehört zu den bedeutendsten Gegenständen der sozialwissenschaftlichen Erforschung des Glücksspiels (für einen Überblick vgl. Miyazaki et al. 1998). Fragen der Umverteilung durch Glücksspiel sind soziologisch bedeutsam, wenn die Form der Umverteilung Einfluss auf soziale Ungleichheiten hat. Tatsächlich zeigen viele der vorwiegend in den Vereinigten Staaten durchgeführten Studien, dass Lotterien eine Form der regressiven Besteuerung sind. Untere Einkommensbezieher verausgaben einen höheren Anteil ihres Einkommens für Lotterielose als Bezieher höherer Einkommen. Für Deutschland ist diese Frage bislang nicht untersucht worden (Tolkemitt 2002: 155).

Wir knüpfen mit diesem Beitrag an die internationale Diskussion zur Verteilungswirkung des Lotteriespiels an, indem wir für Deutschland untersuchen, von welchen soziodemografischen Gruppen die Lotterielosnachfrage, und somit das aus Lotterien erzielte Steueraufkommen, vorwiegend getragen wird. Dabei untersuchen wir mit Mitteln der steuerlichen Inzidenzanalyse die Einkommenselastizität der Lotterielosnachfrage. Im Ergebnis zeigen wir, dass die Höhe des erbrachten Steuerbeitrages unterproportional mit der Höhe des Einkommens variiert. Demnach lassen sich regressive steuerliche Effekte auch für das deutsche Lotto feststellen.

Darüber hinaus untersuchen wir die Verwendungsseite der vom Staat aus dem Lotto erzielten Einnahmen. Zum Teil gehen die nicht ausgeschütteten Anteile der Spieleinsätze unmittelbar in die Länderhaushalte ein. Zum Teil fließen die Einnahmen aus Lottoeinsätzen aber auch zweckgebunden als Direktförderung bestimmten Destinatären aus Breitensport, sozialer Wohlfahrt, Kunst und Kultur zu. Daraus ergibt sich die Frage, ob die Zweckbindung dieser Abgaben die regressiven Belastungswirkungen amortisiert, abfedert oder gar verstärkt. Welche soziodemographischen Gruppen profitieren von diesen Ausgaben besonders? Dazu untersuchen wir die distributionale Inzidenz der Lotterielosbesteuerung, also die Frage, ob diejenigen, welche die Steuern zahlen, auch im gleichen Maße von der Verwendung dieser Gelder profitieren. Am Beispiel der Breitensportförderung zeigen wir, dass die durchschnittliche Nutznießung der Fördermittel sozialstrukturell nur eingeschränkt mit der Gruppe ihrer Beitragszahler übereinstimmt. Die gezielte Verwendung der Beiträge trägt somit zu einer systematischen Verstärkung ungleicher Umverteilungswirkungen durch die Lotterielosbesteuerung bei. Die Frage der Verteilungswirkungen durch die Verwendung von staatlich vereinnahmten Lotteriegeldern ist bislang auch international fast nicht erforscht worden (Rubenstein/Scafidi 2002: 226 f.).

Im ersten Teil des Artikels geben wir einen Überblick über die Struktur des deutschen Glücksspielmarktes mit besonderem Schwerpunkt auf Lotterien. Daran anschließend stellen wir die fiskalische Relevanz des Glücksspiels dar, bevor wir im dritten Teil den Forschungsstand zur Umverteilungswirkung von Lotterien und Erklärungsansätze für die überproportionale Nachfrage unterer sozialer Schichten diskutieren. Im dann folgenden Teil analysieren wir empirisch die in Deutschland zu beobachtenden Umverteilungseffekte durch Lotterien. Die Untersuchung basiert auf den Daten einer für das Forschungsprojekt erhobenen repräsentativen telefonischen Bevölkerungsumfrage (vgl. Abschnitt 5). 


\section{Struktur und Volumen des Glücksspielmarkts}

Die Veranstaltung von Glücksspielen unterliegt in Deutschland einem staatlichen Monopol und ist damit der freien Marktzugänglichkeit entzogen. Das Anbieten von Glücksspielen ist nach $\S 284 \mathrm{ff}$. StGB verboten und steht unter Strafe, sofern nicht eine staatliche Genehmigung der kontrollierten Betreibung vorliegt. Gemäß $§ 1$ des Staatsvertrages zum Lotteriewesen in Deutschland soll damit der „,natürliche Spieltrieb der Bevölkerung in geordnete und überwachte Bahnen" gelenkt werden (vgl. Tettinger/Ennuschat 1999). Aus diesem Kanalisierungsauftrag ergeben sich zwei Konsequenzen für die staatliche Regulierung: Erstens muss durch die Bereitstellung eines kontrollierten Spielangebots die Spielleidenschaft der Bevölkerung angemessen bedient werden. Damit soll einerseits das Aufflammen unkontrollierter Veranstaltungen illegaler Glücksspiele verhindert und deren Attraktivität unterwandert werden. Andererseits soll die staatliche Kontrolle einen ordnungsgemäßen und nachvollziehbaren Ablauf des Glücksspiels sicherstellen und möglichen Spielmanipulationen oder anderen illegalen Begleiterscheinungen wie z.B. der Geldwäsche vorbeugen. Zweitens soll durch die staatliche Monopolisierung des Glücksspiels eine an Gewinnmotiven orientierte Ausbeutung der Spielleidenschaft verhindert werden, indem eine gedämpfte, nicht auf Gewinnmaximierung ausgelegte Bereitstellungsstrategie von Glücksspielangeboten verfolgt wird. Mit dieser Regelung soll das dem Spiel inhärente Gefährdungspotenzial für die Bevölkerung reduziert werden.

Der staatlich konzessionierte legale Glücksspielmarkt umfasst ein Jahresvolumen von derzeit etwa 30,5 Mrd. Euro Bruttoumsatz. ${ }^{1}$ Damit entspricht der Glücksspielmarkt etwa 1,3 Prozent des BIP. Die umsatzstärksten Segmente innerhalb dieses Marktes sind Geldspielautomaten (18 Prozent), Spielbanken (31 Prozent) und der Deutsche LottoToto-Block (27 Prozent). ${ }^{2}$ Innerhalb der Spiele des deutschen Lotto-Toto-Blocks fallen etwa zwei Drittel des Umsatzes auf die Wetteinsätze des Spiels „6 aus 49“, dem klassischen Samstags- und Mittwochslotto. Der aktuelle Jahresumsatz dieses Spiels beziffert sich für das Jahr 2006 auf knapp über fünf Mrd. Euro.

Die Umsätze des Lottos sind seit der ersten Ausspielung im Jahr 1955 stetig gewachsen. Die reale Wachstumsrate liegt bei jährlich 4 Prozent. Abbildung 1 veranschaulicht den preisbereinigten Jahresumsatz des deutschen Lottos von 1955 bis 2006. In der Grafik sind einige ausgewählte Änderungen und Erweiterungen der Spielgestaltung seit 1955 festgehalten. Ersichtlich wird daraus, dass Veränderungen der Ausgestaltung des Produktangebotes für hervorstechende Umsatzsteigerungen verantwortlich sind. So

1 Bemessungszeitraum ist das Jahr 2005 (vgl. SES Research, zit. n. Financial Times Deutschland vom 30.07.2006). Der Netto-Umsatzanteil des deutschen Lotto-Toto-Blocks liegt jedoch weitaus höher, da die einzelnen Glücksspielsegmente mit unterschiedlich hohen Gewinnausschüttungsquoten operieren. Während etwa Geldspielautomaten, Spielcasinos oder Pferdewetten 70 bis 97 Prozent der Spieleinsätze als Gewinne ausbezahlen, liegt die mittlere Ausschüttung der Spiele des Lottoblocks bei 48 Prozent. Berücksichtigt man dieses Datum zur Schätzung des Netto-Marktvolumens der einzelnen Spielsegmente, so verkörpert der deutsche Lotto- und Toto-Block mit schätzungsweise über 50 Prozent Marktanteil den größten Anbieter auf dem deutschen Glücksspielmarkt (Albers 1993: 138 f.; Tolkemitt 2002: 30).

2 Der deutsche Lotto-Toto-Block ist die Dachorganisation der sechzehn Lotteriegesellschaften der Bundesländer in Deutschland. 
Abbildung 1: Realer Umsatz Lotto 1955 - 2006, in Mio Euro; fortschreitende Ausgestaltung des Lottospiels

Inflationsbereinigte

Umsätze

(in Mio. Euro)

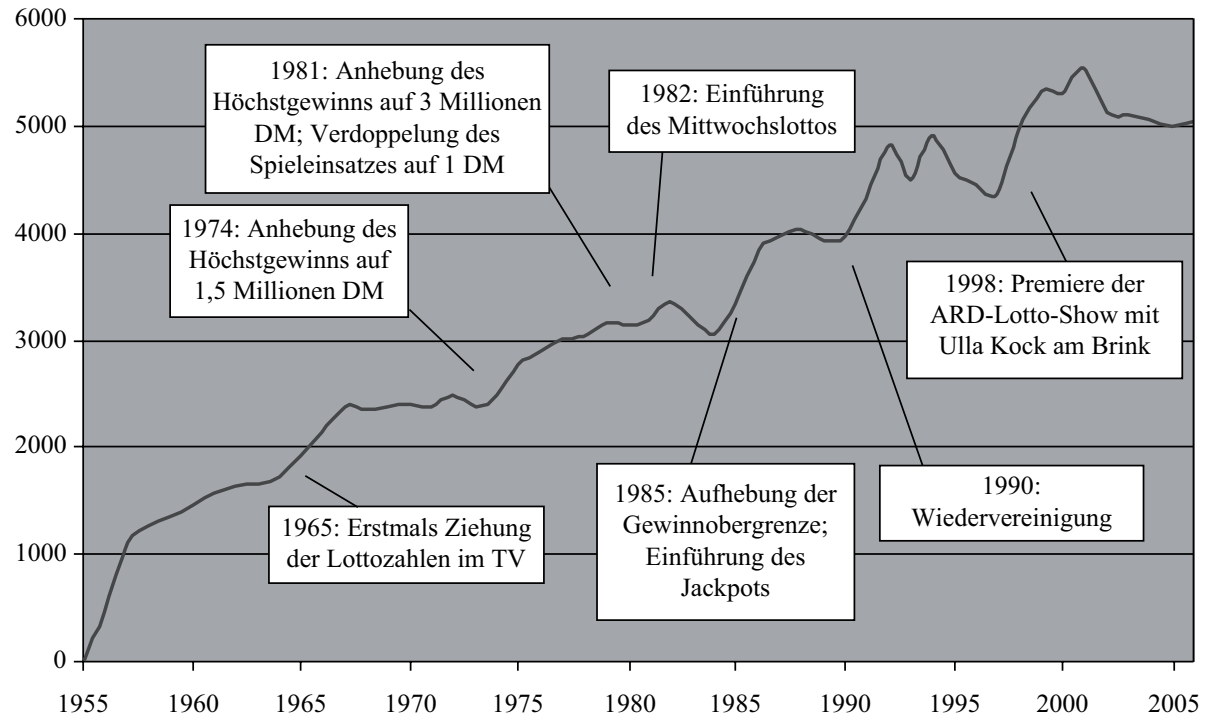

Quellen: Umsatzdaten entstammen dem Lotto-Toto-Archiv, Münster; Inflationsbereinigung nach Preisindizes des Statistischen Bundesamtes, Wiesbaden; historische Informationen aus „50 Jahre Toto-Lotto Baden-Württemberg“, Staatliche Toto-Lotto GmbH Baden-Württemberg, Verlag Kohlhammer, 1998: $202 \mathrm{f}$.

etwa folgen mit der Einführung der Fernseh-Live-Übertragung der Ziehung der Lottozahlen im Jahr 1965, der Anhebung der Hauptgewinnhöchstgrenzen in den Jahren 1974 und 1981, der Verdoppelung des Spieleinsatzes und der anschließenden Aufhebung der Höchstgrenze des Hauptgewinnes und der gleichzeitigen Einführung eines rollierenden Jackpot-Systems in den Jahren 1981 und 1985 bedeutsame Wachstumssprünge.

Gestützt auf ein dichtes Vertriebsnetz von über 26.000 Annahmestellen ist das Lotto das populärste Glücksspiel in Deutschland. Ein Anteil von 40 Prozent der erwachsenen deutschen Wohnbevölkerung spielt innerhalb eines Jahres mindestens einmal Lotto ${ }^{3}$, womit das Lottospiel die mit Abstand höchste Jahresprävalenzrate aller Glücksspiele aufweist. ${ }^{4}$ Unter den Lottospielern beobachten wir zudem eine große Beständigkeit des Spielens: Mehr als jeder zweite Lottospieler spielt regelmäßig mindestens einmal im

3 Sofern nicht anders vermerkt, beruhen die folgenden Angaben zur Prävalenz des Lottospiels auf den Daten unserer Bevölkerungsumfrage. Für eine ausführlichere Darstellung der Struktur des Glücksspielmarktes s. Beckert/Lutter (2007).

4 Die Jahresprävalenzen anderer Glücksspiele liegen unseren Schätzungen zufolge weitaus geringer. Spielbanken werden etwa nur von 4,5 Prozent der Bevölkerung, Geldspielautomaten zu 3,5 Prozent mindestens einmal innerhalb eines Jahres frequentiert. Das nach den Lotterien beliebteste Glücksspiel sind Sportwetten mit einer Jahresprävalenz von 5,8 Prozent. 
Monat, etwa drei Viertel dieser Spieler spielen wöchentlich. Der geschätzte monatliche Durchschnittseinsatz für Lose liegt bei rund 17 Euro, was einen mittleren Anteil von knapp 2 Prozent des verfügbaren Nettoeinkommens der Spieler ausmacht. Die durchschnittliche Einsatzhöhe der Lottospieler variiert dabei nach der Spielfrequenz. So setzen Gelegenheitsspieler etwa 6,1 Euro, monatliche Spielteilnehmer etwa 13,2 Euro und regelmäßige Wochenspieler durchschnittlich 30,1 Euro im Monat für die Teilnahme am Lottospiel ein. Jede Woche vereinnahmen die Annahmestellen ca. 100 Millionen Euro, wovon rund 60 Prozent auf das Samstagslotto entfallen.

\section{Fiskalische Relevanz des Glücksspiels}

Die Summe staatlicher Einnahmen aus Betrieb und Konzessionierung von Glücksspielen liegt derzeit bei rund 5 Mrd. Euro ${ }^{5}$ und ist damit von erheblicher fiskalischer Bedeutung. Dies gilt insbesondere für die Länderhaushalte, wo die Glücksspieleinnahmen einen Anteil von rund 18 Prozent der Ländersteuereinnahmen ausmachen. Insgesamt erzielt der Staat ca. 1,1 Prozent seiner Einnahmen aus dem Glücksspiel. Die Einnahmen aus dem Glücksspiel entsprechen in etwa dem Volumen der Grunderwerbssteuer, dem 1,2-fachen der Erbschaftssteuer oder dem 1,5-fachen der Einnahmen aus alkoholbezogenen Steuern (vgl. Bundesministerium der Finanzen 2006; Deutsche Hauptstelle für Suchtfragen e.V. 2006).

Die Erträge setzen sich aus der Rennwett- und Lotterielossteuer (2005: 1,8 Mrd. Euro) sowie der Spielbankabgabe (2005: 0,563 Mrd. Euro) zusammen und aus Konzessions- sowie Zweckabgaben. Letztere kommen entweder dem Landeshaushalt zugute oder sie werden als zweckgebundene Direktabgabe zivilgesellschaftlichen Empfängergruppen zugewiesen. In der Regel kommen die Direktabgaben der Förderung gemeinnütziger Projekte dem Breitensport, der sozialen Wohlfahrt, Kultureinrichtungen wie Museen und Theatern oder der Pflege von Denkmälern zugute.

Mit einem Steuersatz von 39 Prozent der Lotteriesteuer und den Konzessionsabgaben zusammengenommen sind Lotterielose hoch besteuerte Güter. Dies gilt einmal im Vergleich zur Besteuerung anderer Konsumgüter. So werden die allermeisten Konsumgüter nur mit dem Mehrwertsteuersatz von 7 bzw. 19 Prozent besteuert; Zigaretten werden zusätzlich mit 24,2 Prozent des Verkaufspreises besteuert; für Alkoholika wie Bier, Schaumwein oder Spirituosen liegt die Besteuerung zwischen 3 Prozent und 21 Prozent des durchschnittlichen Verkaufspreises (vgl. Jahrbuch Sucht 2006). ${ }^{6}$ Lediglich

5 Diese Zahl beruht auf einer Hochrechnung aktueller Umsätze auf Basis der Befunde bei Meyer (2006: 121f.), der für 2004 4,2 Mrd. Euro Steuereinnahmen aus Glücksspielen beziffert. Hinzu kommen noch die für das Jahr 2004 angegebenen rund 700 Mio. Euro Steuereinnahmen aus Spieleinsätzen bei Geldspielautomaten. Geldspielautomaten gelten zwar de facto, nicht aber de jure als Glückspiele.

6 Da die Alkoholsteuer sich auf den reinen Alkoholgehalt pro Liter alkoholischen Getränks bezieht, hängt die prozentuale Besteuerung vom Verkaufspreis des Getränks ab. Der angegebene Steuersatz beruht daher auf Annahmen bezüglich des Verkaufspreises. Diesen haben wir für Bier bei 3 Euro pro Liter angesetzt, bei Branntwein und Spirituosen bei 25 Euro pro Liter. 
die Kraftstoffsteuer, die für Benzin bei etwa 66 Prozent des Verkaufspreises liegt, übersteigt die Höhe der Besteuerung von Lotterielosen.

Erst recht ist die Besteuerung von Lotterielosen hoch, wenn man diese als Finanzinvestition versteht (Friedman/Savage 1948; McCaffery 1994). Andere als Investitionen zu verstehende Transaktionen weisen keine oder eine viel geringere Besteuerung auf: So liegt die Grunderwerbssteuer (außer in Berlin) bei 3,5 Prozent des Kaufpreises, bei Finanzmarkttransaktionen werden, mit vielen Einschränkungen, lediglich erzielte Gewinne besteuert, bei Kunstkäufen fällt der ermäßigte Mehrwertssteuersatz an. Die hohe Besteuerung von Lotterielosen lässt sich dabei nicht aus dem staatlichen Auftrag der Bekämpfung der Spielleidenschaft durch Erhöhung des Preises für Lotterielose herleiten - für die effektive Reduzierung der Nachfrage müsste die Spielstruktur geändert werden, z. B. durch sehr viel niedrigere Hauptgewinne -, sondern verweist eindeutig auf die Einnahmeerzielungsabsicht des Staates.

In der Steuerverteilungslehre wird die Güte einer Steuer nach zwei Grundprinzipien beurteilt (vgl. Brümmerhoff 1992; Haller 1981): dem Leistungsfähigkeitsprinzip und dem Äquivalenzprinzip. Das Leistungsfähigkeitsprinzip besagt, dass eine Steuerlast gemäß der wirtschaftlichen Lage ihrer Beitragszahler möglichst gerecht verteilt sein soll. Die wirtschaftliche Lage von Personen wird dabei in der Regel über das Einkommen als Indikator von Vermögen gemessen. Die Höhe einer steuerlichen Last sollte dabei mindestens proportional zur Höhe des Einkommens, im Idealfall jedoch progressiv verlaufen, die aufzubringende Steuerlast also mit steigender wirtschaftlicher Potenz überproportional ansteigen. Umfragen zeigen, dass das Prinzip der Steuerprogression auf eine breite Zustimmung in der Bevölkerung trifft (vgl. Liebig/Mau 2005).

Das Äquivalenzprinzip als zweites Gütekriterium verlangt eine gerechte, an den Präferenzen oder Verbrauchsgewohnheiten der Steuerzahler orientierte Verwendung der Steuermittel. Demnach müssen die Träger der Steuer mit der Art und Weise ihrer Verwendung übereinstimmen oder sie müssen in irgendeiner Form einen Nutzen aus der Verwendung der Gelder ziehen können. So kommen etwa die Einnahmen der Kfz-Steuer in Form von Aufwendungen für den Straßenbau oder der Gewährleistung von Verkehrssicherheit letztlich den Autofahrern als den Zahlern dieser Steuer wieder zugute. Ein Argument für die Erhebung von Tabak- und Alkoholsteuern besteht darin, dass diese den Konsumenten dieser Genussmittel in Form der Deckung ihrer erhöhten Gesundheitskosten wieder zugute kommen. Gemäß diesem Prinzip müssten auch die Lottoeinnahmen den Spielern wieder zugute kommen.

Da den Lotteriesteuereinnahmen jedoch keine für die Lotteriespieler spezifischen Ausgaben gegenüberstehen - diese sind durch die Deckung der Kosten der Durchführung der Lotterie aus den Spielumsätzen bereits berücksichtigt - lässt sich die Steuer nicht aus dem Äquivalenzprinzip legitimieren. Dies gilt zumindest für den Teil der staatlichen Einnahmen aus dem Glücksspiel, die in den allgemeinen Haushalt einfließen. Bei den Konzessions- und Zweckabgaben könnte das Äquivalenzprinzip dann erfüllt sein, wenn die Mittelverwendung mit den Präferenzen und dem tatsächlichen Konsumverhalten der Lotteriespieler korrespondiert.

Für die in den allgemeinen Haushalt eingestellten Anteile der staatlichen Einnahmen aus dem Lotteriespiel müsste das Leistungsfähigkeitsprinzip zur Beurteilung herangezogen werden. D.h. als gerecht wäre diese Steuer dann zu werten, wenn das Steuer- 
aufkommen gemessen am Einkommen der Lotteriespieler mindestens proportional verliefe oder sogar eine Progression dergestalt aufweist, dass höhere Einkommensgruppen zu diesem Steueraufkommen überproportional beitrügen. Ist dies nicht der Fall, ließe sich von einem Umverteilungseffekt durch die staatliche Lotterie sprechen, der keine Entsprechung in den anerkannten Besteuerungsprinzipien fände. Die Besteuerung wäre regressiv.

\section{Forschungsstand}

\subsection{Empirische Ergebnisse zur sozialstrukturellen Wirkung von Lotterien}

Die Frage, welche Umverteilungseffekte Lotterien haben, ist insbesondere für die Vereinigten Staaten untersucht worden. Ein Großteil dieser Studien kommt zu dem Schluss, dass Lotterien eine Form der regressiven Besteuerung sind und damit sozialstrukturell ungleiche Verteilungswirkungen haben. Dieser Befund erweist sich als relativ einheitliches Ergebnis, dessen Robustheit auch durch die Verwendung ganz unterschiedlicher Datentypen und Verfahrensweisen Unterstützung findet. ${ }^{7}$ Vielfältig ist die Art der Berechnung der Regressivität: Einige Studien beschränken sich auf die Darstellung einfacher Assoziationen wie der Untersuchung bivariater Kreuztabellen, in denen der Zusammenhang zwischen dem als Anteil am Einkommen bemessenen Spieleinsatz und dem verfügbaren Nettoeinkommen gemessen wird (vgl. Brinner/Clotfelter 1975). Ein verbreiteter Ansatz ist die Benutzung des Suits-Index, eines steuerlichen Konzentrationsmaßes, das analog dem Gini-Koeffizienten die Ungleichverteilung von Einkommen und Steuerlast zusammenfasst (vgl. Suits 1977b). Nur wenige Studien spezifizieren multivariate Modelle, bei denen die Elastizität des Einkommens mit der Höhe des Steuerbeitrages modelliert wird (vgl. Borg/Mason 1988).

Die Studien unterscheiden sich nicht nur in ihrer Methodik, sondern auch in ihren Schlussfolgerungen hinsichtlich der Stärke regressiver Effekte. ${ }^{8}$ Für Texas belegen Price

7 So untersuchen einige Arbeiten ausschließlich makrostrukturelle Aggregatdaten, indem sie den Zusammenhang zwischen aggregierten Pro-Kopf-Umsätzen und dem Einkommensniveau bestimmter räumlicher Bezirke beleuchten (vgl. Clotfelter 1979; Hansen 1995; Jackson 1994; Mikesell 1989; Vasche 1985). Andere Studien untersuchen die steuerliche Inzidenz des Lottospiels mittels Mikrodaten, zumeist unter Verwendung bevölkerungsrepräsentativer Umfragedaten oder auch unter Verwendung von Daten aus der Befragung von Lotteriegewinnern (Borg/Mason 1988; Brinner/Clotfelter 1975; Clotfelter/Cook 1991; Kitchen/Powells 1991; Livernois 1987; McConkey/Warren 1987; Spiro 1974; Suits 1977a; Vaillancourt/Grignon 1988). Weitere Studien vermischen mikro- und makrostrukturelle Daten wie etwa Clotfelter/ Cook (1987), Koza (1982) oder Heavey (1978), die allesamt Umfragedaten von Lotteriegewinnern nach regionalen Strukturmerkmalen und Umsatzangaben einteilen.

8 Einige wenige Studien konstatieren proportionale oder gar progressive steuerliche Effekte. So findet etwa Mikesell (1989) mit Hilfe von Aggregatdaten für den Staat Illinois ein proportional zum Einkommen variierendes Steueraufkommen aus Lotterieeinsätzen. Jackson (1994) konstatiert in einer Untersuchung der Lotterie im Staate Massachusetts aus dem Jahr 1983 gar Verteilungen von Lotterieeinsätzen, die überproportional zum Einkommen verlaufen. Allerdings berichtet derselbe Autor anhand einer Replikation der Untersuchung mit Daten für das 
und Novak (1999), dass die dortige Lotterielosbesteuerung in ihrer Regressivität weitaus ungleicher variiert als die durchschnittliche Beitragsverteilung der ebenso regressiven Belastung durch die Mehrwertsteuer. Hansen et al. (2000) belegen unter Verwendung zeitbezogener Aggregatdaten für fünf US-amerikanische Bundesstaaten eine in jüngerer Zeit stärkere regressive Inzidenz als frühere Datenquellen für dieselben Bundesstaaten dies andeuten. Zudem scheint es nationale Unterschiede in der Stärke regressiver Effekte zu geben. Untersuchungen in Kanada zeigen, dass kanadische Lotterien eine durchgängig geringere Regressivität als US-amerikanische Lotterien aufweisen (vgl. Kitchen/Powells 1991; Livernois 1987; Vaillancourt/Grignon 1988). Ebenfalls variieren die Befunde zwischen einzelnen Spielen. So sind tägliche Lotterien oder Rubbelloslotterien stärker regressiv im Vergleich zu dem klassischen Lotto oder den Klassenlotterien (vgl. Clotfelter/Cook 1991; Mikesell 1989; Price/Novak 1999).

In weit geringerem Maß wird in der Forschungsliteratur die Frage der Verteilungswirkungen der Verwendung der eingenommenen Gelder untersucht. Wenn Bezieher geringer Einkommen nicht nur einen höheren Anteil ihres Einkommens für Lotterielose ausgeben, sondern diese auch noch in geringerem Maß von den Verwendungen der staatlich vereinnahmten Lottomittel profitieren, etwa weil Lottospieler weniger häufig die Güter nachfragen, die durch Lottomittel gefördert werden, verstärkt sich die Regressivität. ${ }^{9}$

Borg und Mason (1988) setzen den pro Haushalt für den Staat Illinois geschätzten Nutzen eines aus Lotteriegeldern subventionierten Bildungsprogramms in Beziehung mit den pro Haushalt verausgabten Lotteriespieleinsätzen und schlussfolgern, dass die Art der Zweckbindung der Gelder den Grad regressiver Besteuerung durch Lotterielose zwar vermindert, jedoch von einer gänzlichen Ausgleichwirkung weit entfernt bleibt. Eine Verstärkung regressiver Effekte zeigen Rubenstein und Scafidi (2002) auf, die für Georgia untersuchen, welche Lottospieler Mittel aus einem durch Lotteriemittel finanzierten Stipendienprogramm für den Collegebesuch erhalten. Demnach tragen Haushalte der unteren Einkommensniveaus über ihre Lotterieausgaben zwar signifikant stärker zur Finanzierung dieses Stipendienprogramms bei, gleichzeitig profitieren Haushalte der höheren Einkommens- und Bildungsschichten weit überwiegend von diesen Geldern, da sie überproportional häufig die Stipendien in Anspruch nehmen.

Generell korrespondieren diese Befunde mit der an Lotterien häufig geübten Kritik, diese würden insbesondere sozial benachteiligten Schichten eine Bürde auferlegen (Braidfoot 1985; Clotfelter 2000; Clotfelter/Cook 1991; Cosgrave/Klassen 2001; Husz 2002; Karcher 1989, 1992; Nibert 2000, 2006; Reith 1999). Für Reith (1999: 100) etwa stellt die Lotterie eine vom Staat organisierte, rüde Ausbeutung der Hoffnungen sozial benachteiligter Schichten dar, die aufgrund ihrer Position im kapitalistischen System im Lottospiel auf den einzig möglichen Ausweg hoffen. Tatsächlich zeigen Studien, dass die Lotterielosnachfrage nicht nur mit relativ geringerem Einkommen korreliert, sondern generell mit niedrigeren sozioökonomischen Statuspositionen, also geringerer

Jahr 1990 von einer Umkehrung der früheren Befunde und einem nun deutlich regressiven Verhältnis von Einkommen und Spieleinsätzen.

9 Untersucht wurde diese Frage von Borg/Mason (1988), Borg et al. (1991), Livernois (1987), Rubenstein/Scafidi (2002) sowie Stranahan/Borg (2004). 
Bildung, geringerem Berufstatus und häufigeren Zugehörigkeit zu ethnischen Minoritäten (vgl. Brown et al. 1992; Clotfelter/Cook 1991).

\subsection{Theoretische Herleitungen des schichtenbezogenen Nachfragerverhaltens}

Theorien zur Erklärung der Lotterielosnachfrage thematisieren häufig den Zusammenhang zwischen Nachfragerverhalten und Schichtzugehörigkeit, wobei fast durchweg angenommen wird, dass das Lottospiel besondere Faszination auf untere soziale Schichten ausübt. So erklären beispielsweise kognitionspsychologische Ansätze die Glücksspielteilnahme aus dem begrenzten kognitiven Leistungsvermögen der Spieler, die dazu neigen, die sehr niedrigen Gewinnchancen des Lottospiels systematisch zu überschätzen (vgl. Kahneman/Tversky 1979; Meyer/Bachmann 2005; Rogers 1998; Rogers/Webley 2001). Angenommen wird, dass die Verkennung der statistischen Regeln des Spiels generell mit einem geringeren Bildungs- bzw. Statusniveau der Spieler korrespondiert. Empirische Studien zeigen in der Tat einen Zusammenhang zwischen geringer Bildung bzw. Status und der verzerrt positiven Gewinnwahrnehmung des Lotteriehauptpreises auf (Beckert/Lutter 2007: 248; Nibert 2006: 324). ${ }^{10}$

Funktionalistischen Theorien des Spannungsmanagements zufolge ist die Lotterielosnachfrage ebenfalls schichtspezifisch. Die Spielbeteiligung wird aus der Ventilfunktion des Glücksspiels erklärt, mit dem die Spieler den in modernen Gesellschaften inhärenten strukturellen Spannungen zu entrinnen versuchen (vgl. Bloch 1951; Devereux 1980; Frey 1984). Die anomietheoretische Variante dieser Theorie postuliert, dass Personen, die Statusinkonsistenzen verspüren, in erhöhtem Maße Lotto spielen, weil mit dem Lotteriegewinn die Hoffnung auf soziale Aufwärtskatapultierung verknüpft ist. Demnach ist insbesondere bei den unteren Mittelschichten eine verstärkte Spielbeteiligung zu erwarten, da diese mehr als andere Schichten sich in einem spannungsgeladenen Statusgefüge zwischen hohen aufwärtsgerichteten Aspirationen sozialer Mobilität und starken Einschränkungen in den Möglichkeiten des sozialen Aufstiegs befinden (vgl. Frey 1984; Tec 1964). Auch diese Annahme findet empirische Bestätigung (vgl. Beckert/Lutter 2007; Downes et al. 1976; King 1985; Smith et al. 1976). Akteure neigen außerdem dazu, in verzweifelten finanziellen Lagen Lotterielose verstärkt nachzufragen, in der Hoffnung, ihre Situation durch den möglichen Gewinn radikal wenden zu können. Da untere soziale Schichten eine größere Wahrscheinlichkeit haben, in solche Lebenssituationen zu geraten, liegt hierin ein zusätzliches schichtbezogenes Moment des Nachfragerverhaltens (vgl. Blalock et al. 2007).

Vermutet wird außerdem, dass die stärkere Faszination des Lottospiels für untere soziale Schichten eine Erklärung in der völligen Gleichheit der Gewinnchancen findet (McCaffery 1994: 88). Nicht persönliche Leistung, Talent, Kreativität oder soziales und kulturelles Kapital bestimmen den Spielerfolg, sondern allein der Zufall. Diese Chancen-

10 Hieraus ließen sich auch die jüngst vom Bundesverfassungsgericht geforderten Auflagen an die Lotteriegesellschaften ableiten, zur Einhaltung des gesetzlichen Kanalisierungsauftrages in der Bewerbung des Spiels auf eine verzerrte Darstellung der Gewinnchancen zu verzichten und die Spieler über die tatsächlichen Gewinnwahrscheinlichkeiten stärker aufzuklären. Allerdings zeigt sich empirisch, dass für die überwiegende Mehrheit von Lottospielern keine verzerrten Gewinneinschätzungen festzustellen sind (vgl. Beckert/Lutter 2007: 247). 
verteilung unterscheidet sich von sämtlichen anderen Verteilungssituationen in modernen Gesellschaften. Für Angehörige unterer sozialer Schichten hat die egalitäre Chancenverteilung eine größere Attraktivität, weil im Vergleich mit meritokratischen oder askriptiv geprägten Verteilungssituationen die Chancen, ,zu den Gewinnern“ zu gehören, relativ besser sind. Umgekehrt verhält es sich für die oberen sozialen Schichten.

Schließlich lässt sich die größere Attraktivität des Spiels für untere soziale Schichten aus den schichtenabhängigen Konsummöglichkeiten erklären. Neuere soziologische Konsumtheorien erklären die Nachfrage nach Konsumgütern in funktional gesättigten Märkten aus der durch den Erwerb von Konsumgütern ausgelösten Evokation von Phantasievorstellungen (vgl. Campbell 1987). Das Konsumgut induziert Fantasien über die Realisierung materieller Träume und die damit verbundene gesteigerte soziale Anerkennung. Für Lotterielose gilt dies in besonderem Maße. Zwei Drittel aller Lottospieler stellen sich vor, was sie mit dem möglichen Gewinn machen würden (Beckert/Lutter 2007: 266). Während diese lottoinduzierten Phantasiewelten „billig“ zu haben sind, sind untere soziale Schichten von den meisten anderen „evokativen Konsumgütern“ ausgeschlossen. Hierzu zählen teure Positionsgüter wie exquisite Kleidung, erlesene Weine oder luxuriöse Automobile (vgl. Cohen 2001: 730 ff.). ${ }^{11}$ Untere soziale Schichten werden aufgrund ihres relativen Ausschlusses von anderen Konsummöglichkeiten stärker auf Lotterielose gelenkt als Angehörige höherer sozialer Schichten (Blalock et al. 2007).

Die vorhandene Forschungsliteratur zu den Verteilungswirkungen des Lotteriespiels weist überwiegend auf regressive Effekte hin. Die angeführten theoretischen Ansätze können diese empirisch zu beobachtende Nachfrageselektion, die trotz der hohen allgemeinen Verbreitung des Spiels besteht, plausibel herleiten. Inwiefern lassen sich die in der internationalen Literatur festgestellten regressiven Verteilungswirkungen des Lottos auch für Deutschland bestätigen? Zur Beantwortung dieser Frage werden wir im Folgenden zwei Hypothesen empirisch prüfen. ${ }^{12}$ Die erste These bezieht sich auf die Spielbeteiligung und orientiert sich normativ am Besteuerungsprinzip der Leistungsfähigkeit:

Hypothese 1: Lotterielose sind eine regressive Form der Besteuerung, da sie relativ zu ihrem Einkommen überproportional von den Beziehern niedriger Einkommen nachgefragt werden.

Die zweite Hypothese bezieht sich auf die Verwendung der durch das Glücksspiel vereinnahmten Abgaben und orientiert sich normativ am Äquivalenzprinzip:

Hypothese 2: Die Verwendung der aus den Einsätzen der Lottospieler erzielten Gelder gleicht die regressiven Effekte nicht aus, da Lotteriespieler nicht stärker von den Geldern oder Projekten, die durch Lotteriegelder gefördert werden, profitieren als andere.

11 Unter Positionsgütern verstehen wir hier Güter, die einen hohen sozialen Status anzeigen.

12 In der relevanten Forschungsliteratur existieren noch mindestens zwei weitere Hypothesen, deren Überprüfung lohnenswert wäre, jedoch im Rahmen dieser Arbeit nicht näher beleuchtet werden können (vgl. Miyazaki et al. 1998: 162): (1) Die Erwirtschaftung staatlicher Einnahmen über Lotterien ist ökonomisch ineffizient (vgl. Borg/Mason 1988; Brinner/Clotfelter 1975); (2) Die Förderung bestimmter Projekte durch Lotteriegelder ist keine Ergänzung, sondern führt zur Ersetzung der Förderung durch andere Quellen (vgl. Spindler 1995). 


\section{Daten und Methode}

\subsection{Stichprobe}

Für die Prüfung beider Hypothesen verwenden wir Primärdaten aus einer im Auftrag des Kölner Max-Planck-Instituts für Gesellschaftsforschung erhobenen telefonischen Bevölkerungsumfrage über das Nachfrageverhalten von Lotteriespielern in Deutschland. Die Feldarbeiten übernahm im Frühjahr 2006 ein mit der Durchführung beauftragtes sozialwissenschaftliches Umfrageinstitut (SUZ, Duisburg). Im Vorfeld der Erhebung entwickelten wir ein Befragungsinstrument, das wir in mehreren Stufen einer Qualitätsprüfung unterzogen. Dies geschah etwa durch qualitative Gruppendiskussionen einer Gruppe von Lottospielern, Begutachtungen durch Experten der Umfrageforschung (u. a. das ZUMA in Mannheim) sowie zweier Pretests im Feld. Zur definierten Grundgesamtheit der Erhebung zählen wir alle Personen über 18 Jahre der deutschen Wohnbevölkerung aus Haushalten mit einem Telefonanschluss. Für die Stichprobenauswahl, realisiert nach dem Verfahren von Gabler und Häder (1997), das unter Berücksichtigung der im Telefonbuch nichteingetragenen Haushalte eine einfache Zufallsstichprobe der definierten Grundgesamtheit erzeugt, wählten wir einen disproportional geschichteten Stichprobenansatz, bei dem wir Lottospieler im Verhältnis zu ihrer Vorkommenshäufigkeit in der Gesamtheit etwa um das 1,66-fache überrepräsentiert für die Stichprobe auswählten. ${ }^{13}$ Als Lottospieler definieren wir jenen Anteil in der Bevölkerung, welcher mindestens einmal innerhalb des letzten Jahres Lotto gespielt hat. Insgesamt wurden für die Hauptstichprobe 1508 Interviews realisiert, 1002 mit Lottospielern und 506 Interviews mit Nichtspielern.

\subsection{Konstruktion des Merkmals „Steuerbeitrag“}

Ein zentrales Kriterium der nachfolgenden Inzidenzanalyse bildet die Höhe des individuell geleisteten steuerlichen Beitrages, der aus der Nachfrage von Lotterielosen resultiert. Dieser ergibt sich als konstanter Anteil des innerhalb eines bestimmten Zeitraums verausgabten Spieleinsatzes für Lotterielose. Die Höhe des Spieleinsatzes stellt somit ein zentrales Merkmal unserer Analyse dar, das wir wie folgt erfassen: Die als Lottospieler definierte Zielgruppe befragten wir im Fragebogen zunächst danach, wie häufig sie innerhalb des letzten Jahres an der Ausspielung des Samstags- oder Mittwochslotto teilgenommen haben. Aus den drei vorgegebenen Antwortkategorien „,regelmäßig mindestens einmal in der Woche“, „mindestens einmal im Monat“ sowie „nur einige wenige Male im Jahr" leiten wir nun die Anzahl der teilgenommenen Spieltage im Jahr $\mathrm{ab}$, indem wir der ersten Ausprägung den Wert 52 für die durchschnittliche Teilnahme an mindestens 52 Spieltagen im Jahr zuweisen, der zweiten Kategorie den Wert 18 und

13 Für alle Analysen, die sich auf die Gesamtebene beziehen, verwenden wir Gewichtungsfaktoren, die diese stichprobenbedingte Disproportionalität kompensieren. Das zur Gewichtung benötigte wirkliche Verhältnis von Lottospielern zu Nichtspielern in der Bevölkerung schätzten wir zuvor mittels einer größeren Preteststichprobe vom Umfang $n=200$, bei der auch ein standardisiertes kognitives Evaluationsverfahren für das Befragungsinstrument nach der Methode von Faulbaum et al. (2003) zum Einsatz kam. 
der dritten Kategorie den Wert 8 für durchschnittlich 18 respektive 8 teilgenommene Spieltage innerhalb der letzten zwölf Monate. Zugleich befragten wir die Spieler nach der Länge der Laufzeit der von ihnen in der Regel ausgefüllten Spielscheine und nach der Höhe des pro Spielteilnahme durchschnittlich verausgabten Bruttobetrages. Über den mit der Anzahl der teilgenommenen Spieltage im Jahr gewichteten Quotienten aus durchschnittlicher Laufzeit und Betragshöhe pro Spielteilnahme erhalten wir eine als valide anzusehende Verteilung des geschätzten durchschnittlichen Jahreseinsatzes der Lottospieler in Deutschland. ${ }^{14}$

Ein weiteres zentrales Merkmal der nachfolgenden Analyse bildet das Einkommen, weshalb wir das Zustandekommen auch dieses Merkmals kurz erläutern möchten. Allgemein ist die Messung des Einkommens in Umfragen nicht nur mit dem Problem systematischer Fehlangaben behaftet, sondern besitzt als sensible Frage generell hohe, z. T. systematisch verlaufende Verweigerungsquoten (vgl. Groves et al. 2002). In unserem Befragungsinstrument wurde das Einkommen analog zu den Empfehlungen des Statistischen Bundesamtes (2004) zweistufig abgefragt. Zunächst wurde in einer offenen Abfrage das monatlich verfügbare Haushaltsnettoeinkommen, also das Einkommen nach Abzug sämtlicher fixer Kosten wie Steuern, Miete etc. ermittelt. Ein-Personen-Haushalte wurden hierbei direkt nach ihrem persönlichen monatlichen Nettoeinkommen befragt. Sofern nach dieser ersten offenen Abfrage die Zielperson die Einkommensangabe verweigerte, wurde in einer zweiten Abfrage unter besonderem Hinweis der Anonymität die Frage erneut, diesmal jedoch mit vorgegebenen Einkommensklassen gestellt, von denen für die anschließende Fusion beider Stufen die Klassenmitten als bestmögliche Annäherungswerte an die tatsächlichen Werte zur Verwendung kamen. ${ }^{15} \mathrm{Um}$ darüber hinaus ein möglichst realistisches Bild des zur Verfügung stehenden Einkommens zu gewinnen und den Bedarfsunterschieden verschiedener Haushaltsstrukturen gerecht $\mathrm{zu}$ werden, wird aus der zusammengefassten Einkommensvariablen das bedarfsgewichtete Pro-Kopf-Haushaltsnettoeinkommen nach dem aktuellen Vorschlag der OECD berechnet (vgl. Klein 2005: 339 f.).

\section{Resultate}

\subsection{Wer zahlt die Steuer?}

Im folgenden Abschnitt gehen wir der Frage nach, wie sich das Steueraufkommen gemäß dem Leistungsfähigkeitsprinzip nach der wirtschaftlichen Lage ihrer Beitragszahler verteilt. Eine erste deskriptive Annäherung aus den Daten unserer Umfrage verdeutlichen

14 Die auf diesem Wege erlangte Schätzung des Spieleinsatzes erweist sich als valide, wenn man die amtlichen Jahresumsatzangaben der Lotteriegesellschaften als Validierungskriterium heranzieht. Der aus den Stichprobenangaben hochgerechnete Betrag summiert sich auf einen jährlichen Spieleinsatz von 4,9 Mrd. Euro, während die tatsächliche Spieleinsatzsumme von den Lotteriegesellschaften auf etwa 5 Mrd. Euro für das Jahr 2005 beziffert ist. Der Stichprobenwert unterschätzt damit nur geringfügig den tatsächlichen Spielumsatz.

15 Durch diese zweistufige Vorgehensweise konnte bei den Einkommensangaben eine Reduktion der Ausfallquote von 39 auf 18 Prozent erreicht werden. 
die in Abbildung 2 abgetragenen absoluten wie relativ am Einkommen bemessenen mittleren monatlichen Spielausgaben für die fünf Einkommensquintile des monatlich verfügbaren, bedarfsgewichteten Nettoeinkommens. Es zeigt sich dabei ein diametrales Muster beider Größen. Während der absolut bemessene Spieleinsatz mit Zunahme des Einkommens nahezu monoton ansteigt, nimmt der relativ am Einkommen bemessene Spieleinsatz mit steigendem Einkommen sehr deutlich ab.

Eine weitere Möglichkeit der deskriptiven Erfassung von Abweichungen vom Leistungsfähigkeitsprinzip bietet die Verwendung des Indexes nach Suits (1977b), ein Konzentrationsmaß, das den Grad der Regressivität der Lotterielossteuer auf Basis der Abweichung des Flächenanteils unterhalb einer der Lorenzkurve entlehnten Funktion von dem Flächenanteil ihrer Diagonalen zusammenfasst. Die Lorenzkurve bemisst sich aus der zweidimensionalen Darstellung des kumulierten relativen bzw. prozentualen Anteils des Nettoeinkommens am Gesamteinkommen einer Population von Lottospielern und des kumulierten Anteils der Spielausgaben für Lotterielose - als Schätzung der erbrachten Steuerabgaben - am Gesamtspieleinsatz. Die Maßzahl ist analog dem Gini-Koeffizienten definiert als das Verhältnis $S=1-\mathrm{L} / \mathrm{K}$ mit $-1 \leq \mathrm{S} \leq 1$, wobei $\mathrm{L}$ die Fläche unterhalb der auf diese Art erstellten Lorenzkurve angibt und $\mathrm{K}$ die Fläche unterhalb der Diagonalen abbildet, die für perfekte steuerliche Proportionalität steht. Im Fall extremer Progressivität, nämlich dann, wenn die gesamte Steuerlast von den oberen Einkommenssegmenten getragen wird, liegt der Wert bei +1 , wohingegen der Wert 0 eine proportionale Steuer und Werte unterhalb 0 eine regressive Steuerverteilung kennzeichnen. Unserer Berechnung zufolge liefert der Index einen Wert von -0,23, was eine deutlich einkommensungleiche steuerliche Verteilung der Lotterielosnachfrage indiziert.

Abbildung 2: Absolute und relativ zum Einkommen gemessene durchschnittliche monatliche Spielausgaben für Lotto nach Einkommensquintilen

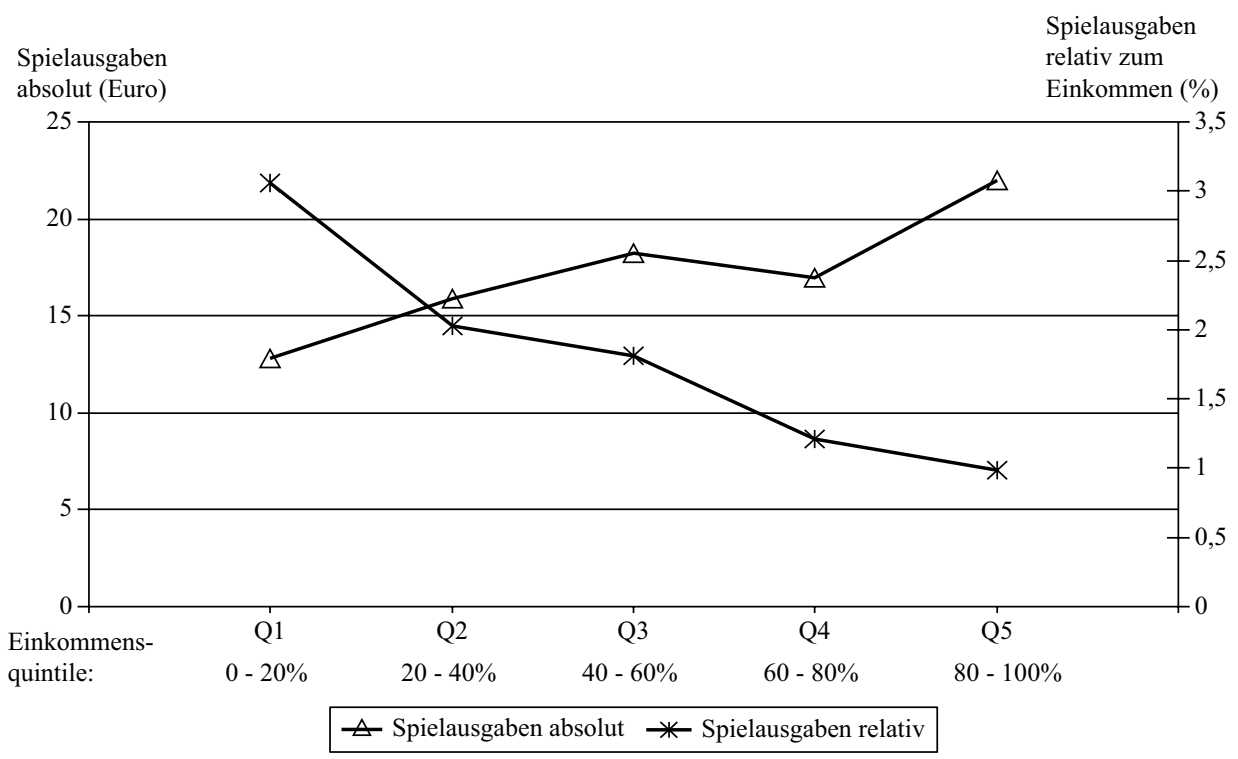


Abbildung 3: Lorenzkurve der steuerlichen Konzentration nach Einkommen; Suits-Index

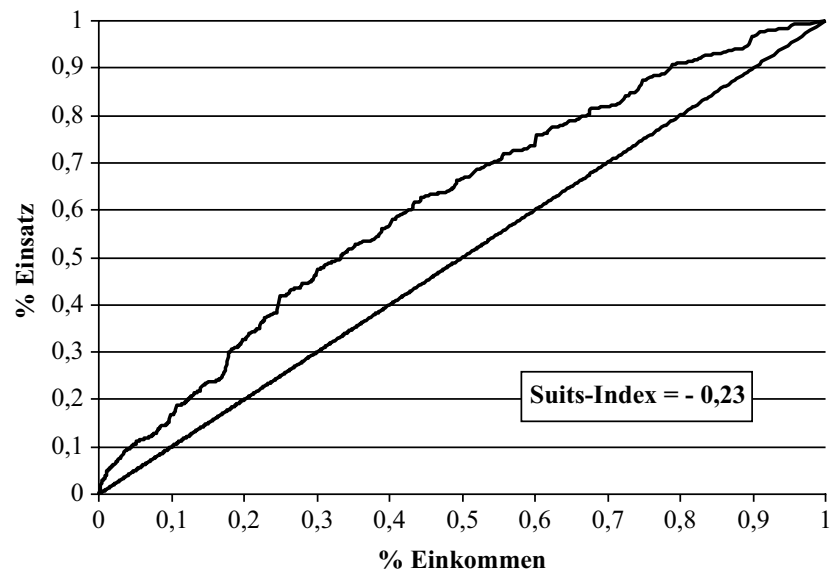

In Abbildung 3 ist dieser Zusammenhang grafisch dargestellt. Die oberhalb der idealtypischen Diagonalen verlaufende Kurve illustriert das regressive steuerliche Verhältnis.

Der Index bietet einige leicht zu erkennende Vorteile, was möglicherweise ein Grund dafür ist, warum ein Großteil der genannten Studien zur Untersuchung der Regressivität sich ausschließlich auf diesen verlässt. Der Koeffizient ist anschaulich zu interpretieren und erlaubt über die graphische Darstellung intuitive Schlussfolgerungen. Ebenso eignet sich die Maßzahl als Vergleichswert, um die Befunde anderer Studien oder Regressivitätswerte anderer Glücksspiele miteinander zu vergleichen (vgl. Suits 1977b).

Dennoch bestehen einige gravierende Nachteile dieser Maßzahl (zur Kritik vgl. Calmus 1981). Gleich dem Gini-Maß ist der Suits-Index nämlich von der Feinabstufung der verwendeten Eingangsdaten abhängig. So müssen Aussagen, die auf einem Vergleich dieser Größe beruhen, dann mit Bedacht interpretiert werden, wenn die Berechnung der Maßzahl auf Basis graduell unterschiedlich skalierter Werte erfolgt. Dass dies zwischen den meisten der in Frage kommenden Studien der Fall ist, zeigt allein die unterschiedliche Datenvielfalt der genannten Studien. Ein weiterer Nachteil besteht unverkennbar darin, dass ausschließlich die Verteilung zweier Merkmale, Spieleinsatz und Einkommen, gemeinsam betrachtet werden. So kann die Kontrolle möglicher Drittvariableneffekte nicht berücksichtigt werden. Tatsächlich konstatieren Hansen et al. (2000: 195 f.) sowie Borg und Mason (1988: 77) für ihre Untersuchungen substanzielle Unterschiede zwischen Befunden, die auf Basis bivariater Inzidenzanalysen beruhen und multivariaten Modellen, die mögliche Drittvariableneffekte konstant halten. Es müssen daher Studien, die sich nur auf diese bivariate Untersuchung der Regressivität verlassen, in ihren Befunden mit Vorsicht betrachtet werden.

Eine wesentlich differenzierte Möglichkeit der Regressivitätsanalyse liegt daher in der Schätzung eines multivariaten Modells. Ein Maß der Regressivität ergibt sich nämlich auch über die Schätzung der Einkommenselastizität der Lotterielosnachfrage, die unter multivariater Konstanthaltung diverser Kovariaten ermittelt werden kann. Allgemein ergeben sich Elastizitäten als Regressionskoeffizienten einer doppelt log-linearen 
Modellspezifikation (vgl. Gujarati 2003: 175 f.). Zur Bestimmung der Einkommenselastizität werden wir im Folgenden die logarithmierte Spieleinsatzhöhe - als Proxy des erbrachten Steueraufkommens - als Linearkombination des logarithmierten Nettoeinkommens unter Berücksichtigung gängiger soziodemografischer Kovariaten schätzen. Der Regressionskoeffizient des logarithmierten Einkommens bildet dann einen Punktschätzer der Einkommenselastizität. Liegt der Koeffizient unter Eins, so kann die Steuer als regressiv bezeichnet werden, da das Steueraufkommen in diesem Fall unterproportional stark mit dem Einkommen wächst. Liegt der Koeffizient bei Eins, so steigt die Steuerlast proportional zum Einkommen, liegt der Wert aber oberhalb von Eins, so handelt es sich um eine progressiv zum Einkommen erbrachte Steuerlast.

Insgesamt schätzen wir die Anpassung dreier Modelle: Erstens bestimmen wir in einem Logit-Modell die soziodemografischen Determinanten der binären Entscheidung, einmal oder häufiger innerhalb des letzten Jahres Lotto gespielt zu haben. Mit diesem Modell erhalten wir einen Einblick in die soziale Rekrutierung der Gruppe der Lottospieler, da es zeigt, in welchen sozialstrukturellen Charakteristika Unterschiede zwischen Spielern und Nichtspielern zu beobachten sind. Zweitens schätzen wir die Höhe der durchschnittlich im Monat verausgabten Spieleinsätze als Funktion soziostruktureller Größen. Wir verwenden dabei je zwei log-lineare Modellspezifikationen, in denen wir zuerst die Höhe der Spielausgaben absolut und im Anschluss in logarithmierter Form als abhängiges Merkmal einbeziehen.

Neben dem (logarithmierten) Einkommen als zentralem Kriterium zur Begutachtung der Regressivität verwenden wir die folgenden soziodemografischen Merkmale als Kovariaten: das Geschlecht (mit Frauen als Referenzkategorie), das Lebensalter (in Jahren), das Bildungsniveau (fünfstufig, mit Hochschulabschluss als höchste Kategorie), Partnerschaft (mit 1 für „mit einem Partner zusammen lebend“; 0 = sonst), Urbanität (mit 1 für Regionen mit mehr als 150000 Einwohnern; $0=$ sonst) und Staatsangehörigkeit (mit 1 für „deutsche Staatsangehörigkeit“ und $0=$ sonst). Das Lebensalter modellieren wir zusätzlich in quadratischer Form als kurvilineare Anpassung an die Beobachtungswerte, da einige Befunde aus amerikanischen Studien auf eine höhere Wahrscheinlichkeit der Lotterielosnachfrage im mittleren Alter hindeuten (vgl. Clotfelter/Cook 1991: 97; Scott/Garen 1994: 132). ${ }^{16}$ Da darüber hinaus die Höhe der Spielausgaben stark mit der Häufigkeit der Spielteilnahme kovariiert, kontrollieren wir in den Modellen zur Schätzung der Höhe der Spielausgaben zusätzlich für die geschätzte Anzahl der Spieltage im Jahr.

Tabelle 1 dokumentiert die Ergebnisse der multivariaten Modellschätzungen. Es handelt sich bei jedem der drei Schätzungen um unterschiedliche Spezifikationen der Funktionsform, weshalb die Koeffizienten in den drei Spalten in unterschiedlicher Weise zu interpretieren sind. Alle Koeffizienten repräsentieren dabei die unstandardisierte Lösung der Schätzergebnisse. In der ersten Spalte finden sich die Effektkoeffizienten, die „odds ratios“ (vgl. Long 1997: 79 f.) des binären Logit-Modells auf das Wahr-

16 Die Spezifikation erfolgt nur für die Schätzung der Spielteilnahme. Für die Höhe der Spielausgaben verzichten wir auf den quadratischen Term, da Voranalysen diesen als nicht signifikant ausgewiesen haben und die Modellanpassung, gemessen am adjustierten R-Quadrat, sich dadurch geringfügig verschlechtert. 
scheinlichkeitsverhältnis (,odds“) der Entscheidung Spielteilnahme vs. Nichtspiel. In den zwei folgenden Spalten sind die Koeffizienten der unterschiedlich spezifizierten OLS-Regressionen abgedruckt, die allesamt den Einfluss sozioökonomischer Merkmale auf die Höhe der Spielausgaben für die Gruppe der Lottospieler beschreiben. ${ }^{17}$ In der zweiten Spalte finden sich die Schätzergebnisse der OLS-Regression auf die Höhe der absoluten Spielausgaben aufgelistet und die dritte Spalte berichtet die Ergebnisse der doppelt-loglinearen Spezifikation. Während hierbei der Regressionskoeffizient des logarithmierten Einkommens nun als Schätzer der Elastizität als ein Maß für die Stärke der Regressivität interpretiert werden kann, beschreiben die übrigen Koeffizienten jeweils die relative Veränderung der Höhe der Spielausgaben bei absolutem Anstieg ihrer Einflussgrößen.

Bevor wir auf das Einkommen zu sprechen kommen, möchten wir zunächst den Einfluss der soziodemografischen Kovariaten erörtern, die uns Informationen über die soziodemografische Struktur der Lotterielosnachfrage liefern. Als eine der wichtigsten Determinanten sowohl für die Spielwahrscheinlichkeit als auch für die Höhe der Ausgaben erweist sich das Bildungsniveau. So sinken mit jeder Zunahme auf der in fünf Stufen erhobenen Bildungsskala die Teilnahmewahrscheinlichkeit, die odds der Spielteilnahme um das 0,8-fache, d. h. jeweils um durchschnittlich 20 Prozent (s. den entsprechenden Koeffizienten in Modell 1). In gleichem Maße sinkt für die Gruppe der Lottospieler die Spieleinsatzhöhe um rund 10 Prozent mit jedem Übergang in eine höhere Bildungsstufe (s. Modell 3). Die Lotterielosnachfrage scheint daher sozioökonomisch in erster Linie eine Frage des Bildungsstandes zu sein, da für die ohnehin sich bereits überhäufig durch geringere formale Bildung auszeichnende Gruppe der Lotteriespieler zusätzlich eine Verausgabung deutlich höherer Spieleinsatzbeträge innerhalb dieser Bildungsschichten $\mathrm{zu}$ beobachten ist.

Gleichwohl kann bei der Lotterielosnachfrage sozialstrukturell keinesfalls von einem Phänomen gesprochen werden, das nur sozial abgehängte Schichten betrifft. Hiergegen spricht bereits die hohe Jahresprävalenz der Beteiligung am Lotto, die bei 40 Prozent der erwachsenen Bevölkerung liegt. Es zeigt sich aber auch, dass sich primär nicht etwa prekär Beschäftigte oder Arbeitslose an der Lotterie beteiligen. Deutlich häufiger zählen wir unter den Lottospielern Beschäftigte, die einer regelmäßigen Erwerbstätigkeit vollzeitbeschäftigt nachgehen. Darauf verweist der Koeffizient der Erwerbstätigkeit im Modell zur Schätzung der Teilnahmewahrscheinlichkeit. Gleichwohl gilt dieser Befund nur für die Spielentscheidung. Sofern nämlich die Entscheidung zur Teilnahme einmal getroffen ist, wirkt sich der Status der Erwerbstätigkeit interessanterweise signifikant

17 Einige ökonometrische Studien modellieren die Entscheidung zur Teilnahme und die Höhe der Spielausgaben simultan mittels eines Tobit-Modells oder zweistufig mittels HeckmanKorrektur (vgl. etwa Sawkins/Dickie 2002; Scott/Garen 1994; vgl. Tobin (1958) und Heckman (1979) zur Methodik allgemein). In unserem Ansatz sind wir an der Schätzung der Einflussstruktur beider Prozesse separat interessiert, weshalb wir die Teilnahmeentscheidung mittels Logitmodell auf der einen und die Höhe des Einsatzes mittels OLS-Regression für die Gruppe der Lottospieler auf der anderen Seite als Ereignisse unabhängig voneinander schätzen. Für die Höhe des Spieleinsatzes wollen wir Effekte allein für die Gruppe der Lottospieler schätzen, was eine Heckman-Korrektur entbehrlich macht (vgl. dazu Engelhardt 1999). Diese Vorgehensweise entspricht der bei Livernois (1987) und Stranahan/Borg (1998). 
Tabelle 1: Regressionsmodelle zur sozialstrukturellen Inzidenz der Lotterielosnachfrage

\begin{tabular}{|c|c|c|c|}
\hline & $\begin{array}{c}\text { (1) } \\
\text { Spielteilnahme } \\
\text { (1 = Lottospieler; } \\
0=\text { Nichtspieler) }\end{array}$ & $\begin{array}{l}\text { (2) } \\
\text { Monatlicher } \\
\text { Spieleinsatz } \\
\quad \text { (in } €)\end{array}$ & $\begin{array}{c}\text { (3) } \\
\text { Monatlicher } \\
\text { Spieleinsatz } \\
\text { (in €; logarithmiert) }\end{array}$ \\
\hline Einkommen & 1,205 & $\mathbf{3 , 5 3 7}$ & 0,280 \\
\hline (in Euro; logarithmiert) & $(1,37)$ & $(2,10)^{* *}$ & $(3,95)^{* * *}$ \\
\hline Geschlecht & 1,154 & 1,686 & 0,062 \\
\hline$(1$ = männlich $)$ & $(1,01)$ & $(0,99)$ & $(0,86)$ \\
\hline Alter & 1,087 & $-0,017$ & $-0,003$ \\
\hline (in Jahren) & $(3,24)^{* * *}$ & $(0,25)$ & $(1,19)$ \\
\hline Alter & 0,999 & & \\
\hline (quadriert) & $(2,54)^{* *}$ & & \\
\hline Bildung & $\mathbf{0 , 8 0 0}$ & $-1,719$ & $-0,094$ \\
\hline$(1=$ gering; $5=$ hoch $)$ & $(3,39)^{* * *}$ & $(2,25)^{* *}$ & $(2,94)^{* * *}$ \\
\hline Erwerbstätigkeit & 1,575 & $-3,427$ & $-0,186$ \\
\hline $\begin{array}{l}(1=\text { Vollzeitbeschäftigt; } \\
0=\text { sonst })\end{array}$ & $(2,79)^{* * *}$ & $(1,79)^{*}$ & $(2,32)^{* *}$ \\
\hline Partnerschaft & 1,503 & 0,285 & 0,094 \\
\hline $\begin{array}{l}(1=\text { lebt mit Partner } \\
\text { zus.; } 0=\text { sonst })\end{array}$ & $(2,84)^{* * * *}$ & $(0,16)$ & $(1,26)$ \\
\hline Urbanität & 0,769 & $-2,829$ & $-0,029$ \\
\hline $\begin{array}{l}(1=>150.000 \text { Einw.; } \\
0=\text { sonst })\end{array}$ & $(1,77)^{*}$ & $(1,53)$ & $(0,38)$ \\
\hline Staatsangehörigkeit & 0,575 & $-10,637$ & $-0,307$ \\
\hline$(1=$ deutsch; $0=$ sonst $)$ & $(1,16)$ & $(1,81)^{*}$ & $(1,24)$ \\
\hline Spielteilnahme & & 12,678 & 0,696 \\
\hline $\begin{array}{l}\text { (in Tagen/Jahr; } \\
\text { logarithmiert) }\end{array}$ & & $(12,48)^{* * * *}$ & $(16,31)^{* * *}$ \\
\hline Konstante & & $\begin{array}{r}-28,163 \\
(2,26)^{* *}\end{array}$ & $\begin{array}{l}-1,104 \\
(2,11)^{* *}\end{array}$ \\
\hline$N$ & 1199 & 705 & 705 \\
\hline McFadden & 0,05 & & \\
\hline LR Chi2 & 66,10 & & \\
\hline Adj. R-Quadrat & & 0,23 & 0,33 \\
\hline
\end{tabular}

Signifikanz: ${ }^{*} \mathrm{p}<0,1 ;{ }^{* *} \mathrm{p}<0,05 ;{ }^{* * *} \mathrm{p}<0,01$ (zweiseitiger Test); in Klammern: z-Statistiken.

negativ auf die Höhe der Spielausgaben aus, wie das nun umgekehrte Vorzeichen dieses Koeffizienten in Modell 2 und 3 verdeutlicht. Nunmehr zeigt sich, das zeitlich geringer beschäftigte Lottospieler im Durchschnitt um etwa 19 Prozent höhere Spielbeträge verausgaben als Vollzeitbeschäftigte (s. Modell 3). Während für die Erklärung des letzteren Befundes deprivationstheoretische Argumente naheliegen, so kann selbst für ersteren Befund vermutet werden, dass in Vollzeitbeschäftigungen mit geringeren Handlungsund Aufstiegsautonomien Aspirationen sozialer Mobilität häufiger über das Lottospiel kanalisiert werden (vgl. Beckert/Lutter 2007: 256).

Für die restlichen Merkmale lassen sich noch einige weitere statistisch signifikante Unterschiede erkennen. So erweist sich das Lebensalter im ersten Modell in der kurvi- 
linearen Anpassung als signifikante Einflussgröße der Spielteilnahme. Die quadratische Spezifikation des Terms impliziert dabei einen invers u-förmigen Verlauf des Zusammenhangs von Lebensalter und Spielwahrscheinlichkeit, die zuerst mit steigendem Alter zunimmt und anschließend fällt. Das Maximum der Teilnahmewahrscheinlichkeit von etwa 0,5 errechnet sich aus dem Modell für ein Lebensalter von etwa 63 Jahren, wenn wir den Einfluss aller anderen soziostrukturellen Variablen an ihrem Stichprobenmittelwert konstant halten. Das heißt, dass nahezu jeder Zweite „sozialstrukturell durchschnittliche“ Bundesbürger im Alter zwischen 60 und 65 Jahren Lotto spielt.

$\mathrm{Zu}$ den weiteren signifikanten Determinanten der Spielwahrscheinlichkeit zählen die Merkmale Partnerschaft und Urbanität. Hierbei zeigt sich, dass Lotteriespieler häufiger in Gemeinschaft mit einem Partner zusammenleben und häufiger aus nichturbanen Regionen mit Gemeinden unterhalb von 150.000 Einwohnern stammen. All diese Effekte weisen allerdings im Hinblick auf die Einsatzhöhe keine ausreichende statistische Signifikanz aus, weshalb die Variation des Spieleinsatzes weder durch das Lebensalter noch durch die Merkmale Partnerschaft oder Urbanität einen substanziellen Erklärungsbeitrag erfährt. Darüber hinaus zeigen sich sowohl hinsichtlich der Teilnahmewahrscheinlichkeit als auch hinsichtlich der Spieleinsatzhöhe keine signifikanten Unterschiede im Geschlechterverhältnis. Befunde amerikanischer Studien zeigen, dass sich Angehörige ethnischer Minoritäten in stärkerem Maße an Glücksspielen beteiligen (vgl. Clotfelter/ Cook 1991: 98; Scott/Garen 1994: 132). Ähnliches zeigen auch unsere Daten für die als Approximation der Zugehörigkeit zu ethnischen Minoritäten zu verstehende Variable „Staatsangehörigkeit“. Während diese in der Entscheidung zur Spielteilnahme keinen Unterschied aufzeigt, so zeigt sich im zweiten Modell ein Effekt auf die Spieleinsatzhöhe. Personen ohne deutschen Pass verausgaben durchschnittlich um 10 Euro höhere Einsatzbeträge als Personen mit der deutschen Staatsangehörigkeit, auch wenn dieser Wert aufgrund des Zusammenspiels zu geringer Fallzahlen und dem Auftreten von Ausreißerwerten für diese Gruppe nur bedingte statistische Aussagekraft hat. Demzufolge verliert der Effekt im dritten Modell durch die glättende Wirkung der Logarithmierung der linken Seite der Modellgleichung an statistischer Präzision und liegt nun außerhalb konventioneller Niveaus von maximal 10 Prozent.

Welchen Einfluss jedoch hat das Einkommen als zentrales Merkmal unserer Untersuchung? In der ersten Spalte zeigt sich, dass die Höhe des Einkommens für die Entscheidung der Spielteilnahme statistisch irrelevant ist. Lottospieler verfügen im Durchschnitt nicht über höhere oder geringere Einkommen als Nichtspieler. Liegt allerdings die Entscheidung zur Spielteilnahme vor, dann ist das Einkommen eine bedeutsame Determinante der Spieleinsatzhöhe. Wie der positive Zusammenhang im zweiten Modell zeigt, wachsen die Spielausgaben mit zunehmendem Einkommen signifikant an. Dieser Befund ist auch in anderen Studien belegt (vgl. Kitchen/Powells 1991: 1847 f.), stellt aber kein Argument gegen die These der Regressivität dar, da nicht die absolute Höhe, sondern die relativ am Einkommen bemessene Höhe der Spielausgaben zur Beurteilung der Regressivität entscheidend ist. Dieses Verhältnis lässt sich adäquat nur mittels der log-linearen Modellspezifikation, dargestellt in der dritten Spalte, bemessen. Der dort ersichtliche Koeffizient liegt mit 0,28 bei einer statistischen Fehlertoleranz von $+/-0,14$ auf dem 95 Prozent-Niveau deutlich unterhalb von Eins und indiziert somit eine regressive Einkommenselastizität der Lotterielosnachfrage. Der Wert impliziert, dass ein 
Anstieg des Einkommens um ein Prozent lediglich mit einem Anstieg der Spielausgaben um durchschnittlich 0,28 Prozent korrespondiert. Die geleisteten Steuerbeiträge steigen damit nur unterproportional mit dem Einkommen an, was die These der Regressivität der Lotterielosnachfrage bestätigt.

Vergleichen wir nun die Befunde unserer Regressivitätsanalyse mit den in Tabelle 2 dargestellten Befunden früherer Studien, so liegt der Grad der Regressivität der deutschen Lotterie im Mittelfeld der Studien aus den USA, Kanada und Spanien. Spanien etwa, das einzige weitere europäische Land, für das Informationen vorliegt, zeichnet sich durch eine im Vergleich zu Deutschland leicht stärkere Regressivität aus (vgl. Garvía/Guillén 2007). Die durchgängig stärksten regressiven Verteilungswirkungen finden sich in den USA, während Kanada die geringste Regressivität aller untersuchten Länder aufweist.

\subsection{Wer profitiert von der Steuer?}

Die Ergebnisse des vorherigen Abschnittes bestätigen die Hypothese, dass auf dem deutschen Lottomarkt die Besteuerung eine regressive Verteilungsform annimmt. Wie zugleich gezeigt werden konnte, ist die Lotterielosnachfrage sozialstrukturell in bestimmter Weise, nämlich vornehmlich durch untere Mittelschichten mit geringerem Bildungsniveau, aber doch größtenteils mittleren absoluten Einkommen, determiniert. In der anschließenden Analyse gehen wir der Frage nach, ob die Verwendung der Steuereinnahmen aus dem staatlichen Lotto gemäß dem Äquivalenzprinzip der Gruppe der Lottospieler zugute kommt. Ist dem so, würde die Regressivität auf der Einnahmeseite durch die spezifische Verausgabung abgemildert oder sogar aufgehoben. Ist dies jedoch nicht der Fall, so potenzieren sich die sozialstrukturell ungleichen Verteilungswirkungen der ohnehin bereits durch die Verletzung des Leistungsfähigkeitsprinzips regressiven Besteuerung. Kann also die Art der Verwendung der Gelder aus Lottomitteln die in der ersten Analyse belegten regressiven Effekte des Steuersystems abfedern oder verstärken sich diese Effekte sogar?

Von den staatlichen Lotterieeinnahmen interessiert uns dabei derjenige Teil, der zweckgebunden bestimmten Empfängergruppen zugute kommt. Hier werden Verwendungsentscheidungen getroffen, bei denen die Herkunft der verausgabten Mittel von den Lottospielern in die Entscheidung zur Verwendung mit eingehen müsste. Wenngleich die Organisation der Verwendungsentscheidungen zwischen den einzelnen Bundesländern variiert, fließen in vielen Bundesländern die Konzessionsabgaben zweckgebunden an Empfänger aus den Bereichen Breitensport, Wohlfahrt, Kunst und Kultur. In einigen Bundesländern (Bayern, Mecklenburg-Vorpommern, Hamburg und Sachsen) fließen die Konzessionsabgaben allerdings ohne spezielle Verwendungsverpflichtung in den allgemeinen Haushalt ein. Die Abgaben- und Verwendungsstruktur des deutschen Lotto- und Toto-Blocks ist in Tabelle 3 tabellarisch dargestellt.

$\mathrm{Ob}$ sich Unterschiede zwischen Spielern und Nichtspielern in der Nutznießung subventionierter Bereiche ergeben, soll in der folgenden Analyse geklärt werden, in der wir die distributionale Inzidenz der Lotterielosbesteuerung, und damit Hypothese 2, näher beleuchten. Wir betrachten dazu einen konkreten Bereich, der zweckgebunden durch Lottomittel bedeutsam gefördert wird. Der größte durch Zweckabgaben subventionierte 
Tabelle 2: Zusammenfassung bisheriger Befunde zur Regressivität staatlicher Lotterien

\begin{tabular}{|c|c|c|c|c|c|}
\hline $\mathrm{Nr}$ & Studie & Jahr & Staat & $S$ & $\eta$ \\
\hline 1 & Spiro (1974) & 1972 & USA (PA) & $-0,20$ & 0,21 \\
\hline 2 & Suits (1977) & 1973 & USA & $-0,31$ & \\
\hline 3 & Brinner/Clotfelter (1975) & 1973 & USA (CT, MA, PA) & $-0,44$ & \\
\hline 4 & Koza (1982) & $1971-76$ & USA (MI, NJ, IL, NY) & $-0,38$ & \\
\hline 5 & Clotfelter (1979) & 1978 & USA (MD) & $-0,33$ & \\
\hline 6 & Vaillancourt/Grignon (1988) & 1982 & Kanada & $-0,18$ & 0,69 \\
\hline 7 & Livernois (1987) & 1983 & Kanada (Edmonton, Alberta) & $-0,10$ & 0,72 \\
\hline 8 & Jackson (1994) & 1983 & USA (MA) & & 2,44 \\
\hline 9 & Clotfelter/Cook (1987) & 1984 & USA (MD) & $-0,36$ & \\
\hline 10 & Borg/Mason (1988) & $1984-86$ & USA (IL) & & 0,25 \\
\hline 11 & Kitchen/Powells (1991) & 1986 & Kanada (6 Regionen) & $-0,18$ & 0,77 \\
\hline 12 & Mikesell (1989) & $1985-87$ & USA (IL) & & 1,26 \\
\hline 13 & Jackson (1994) & 1990 & USA (MA) & & 0,49 \\
\hline 14 & Price/Novak (1999) & 1994 & USA (TX) & $-0,21$ & 0,498 \\
\hline 15 & Garvía/Guillén (2007) & 2006 & Spanien & $-0,23$ & 0,18 \\
\hline 16 & Beckert/Lutter (diese Studie) & 2006 & Deutschland & $-0,23$ & 0,28 \\
\hline
\end{tabular}

Anm.: S ist der Index nach Suits (1977a); $\eta$ ist ein Elastizitätenschätzer; (1) Datenbasis: Befragung von Lotteriegewinnern; div. Lotterien kombiniert; Berechnung von S entnommen aus Suits (1977b); $\eta$ stammt aus bivariatem Modell; (2) Datenbasis: nationaler Haushaltssurvey; div. Lotterien kombiniert; (3) Datenbasis: Umfrage; Passive Lotterie; S entspricht Mittelwert dreier Einzelwerte; zit. n. Clotfelter und Cook (1991); (4) Datenbasis: regionale Aggregatdaten kombiniert mit Umfragedaten; Lotto; S entspricht Mittelwert aus vier Einzelwerten; (5) Datenbasis: regionale Aggregatdaten und Umsatzangaben; S entspricht Mittelwert aus zwei Einzelwerten; (6) Datenbasis: nationaler Haushaltssurvey; div. Lotterien kombiniert; (7) Datenbasis: Umfrage in Edmonton, Kanada; div. Lotterien kombiniert; (8) Datenbasis: regionale Aggregatdaten kombiniert mit Umfragedaten; div. Lotterien kombiniert; (9) Datenbasis: Umfragedaten; Lotto; (10) Datenbasis: Befragung von Lotteriegewinnern; div. Lotterien kombiniert; $\eta$ stammt aus multivariatem Modell (11) Datenbasis: nationaler Haushaltssurvey; div. Lotterien kombiniert; $S$ und $\eta$ entspricht Mittelwert aus je sechs Einzelwerten; (12) Datenbasis: regionale Aggregatdaten und Umsatzangaben; div. Lotterien kombiniert; $\eta$ ist statistisch nicht signifikant von 1 verschieden (13) Datenbasis: regionale Aggregatdaten und Umsatzangaben; div. Lotterien kombiniert; (14) Datenbasis: regionale Aggregatdaten und Umsatzangaben; Lotto; (15) Datenbasis: Umfragedaten; div. Lotterien kombiniert.

Bereich ist der des Breitensports, der mit etwa 3 Prozent des Jahresumsatzes und etwa 15 Prozent der Konzessionsabgaben jährlich gefördert wird. ${ }^{18}$ Die Breitensportförderung bietet sich auch deshalb zur genaueren Untersuchung an, weil hier am ehesten vermutet werden kann, dass auch untere Einkommensschichten von der Förderung durch Lottomittel profitieren. Bei Förderung der Hochkultur ist hingegen ohnehin klar, dass diese vornehmlich von hohen und gut gebildeten sozialen Schichten nachgefragt wird.

In welchem Maße sind die Nutznießer der Breitensportförderung aus den Lottoeinnahmen auch - durch ihren Spieleinsatz - die Träger der Kosten? Inwiefern stimmt also die Gruppe der Sportmitglieder mit der Gruppe der Lottospieler sozialstrukturell

18 Bei einer Zahl von etwa 27 Millionen Mitgliedern in deutschen Sportvereinen im Jahr 2005 liegt damit die Pro-Kopf-Förderung aus Zweckabgaben der Lotterien bei rund 6 Euro pro Mitglied im Jahr (Deutscher Sportbund 2005). 


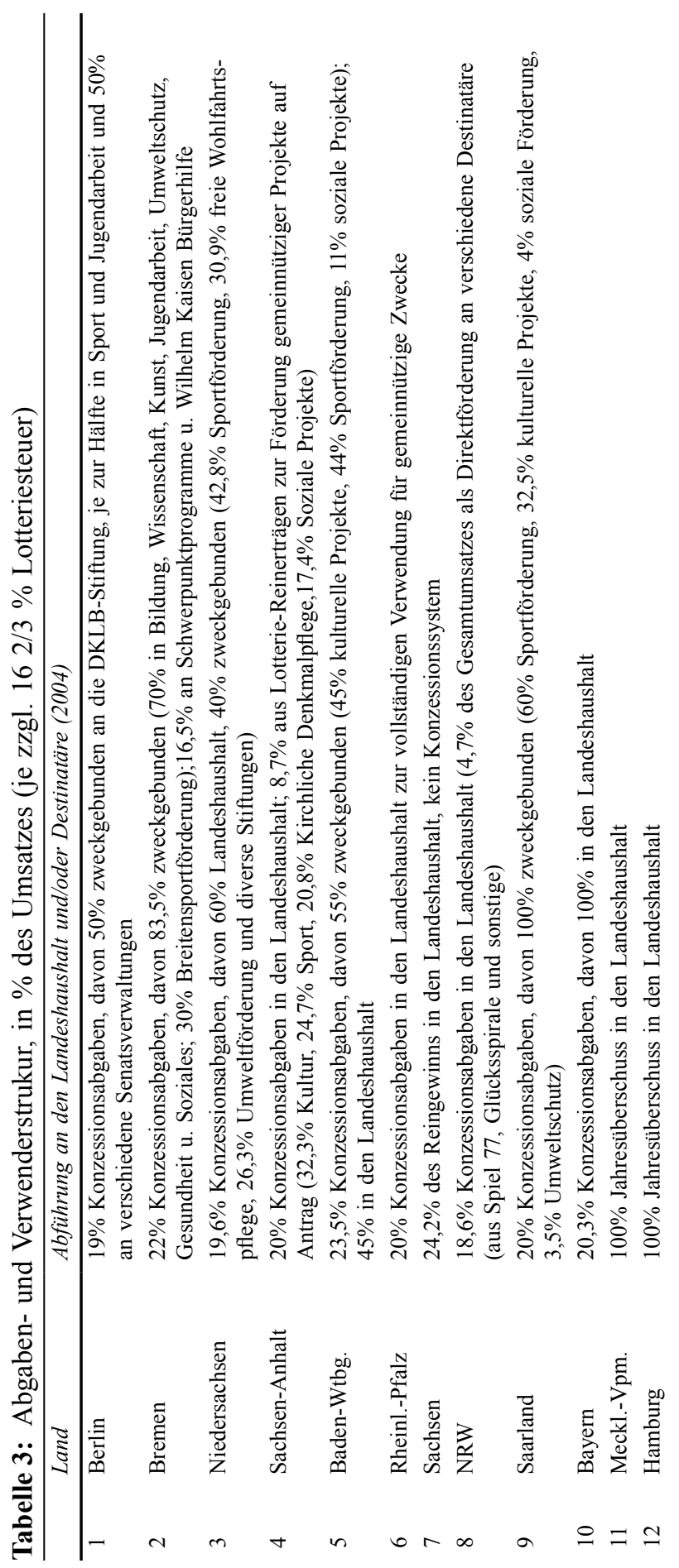




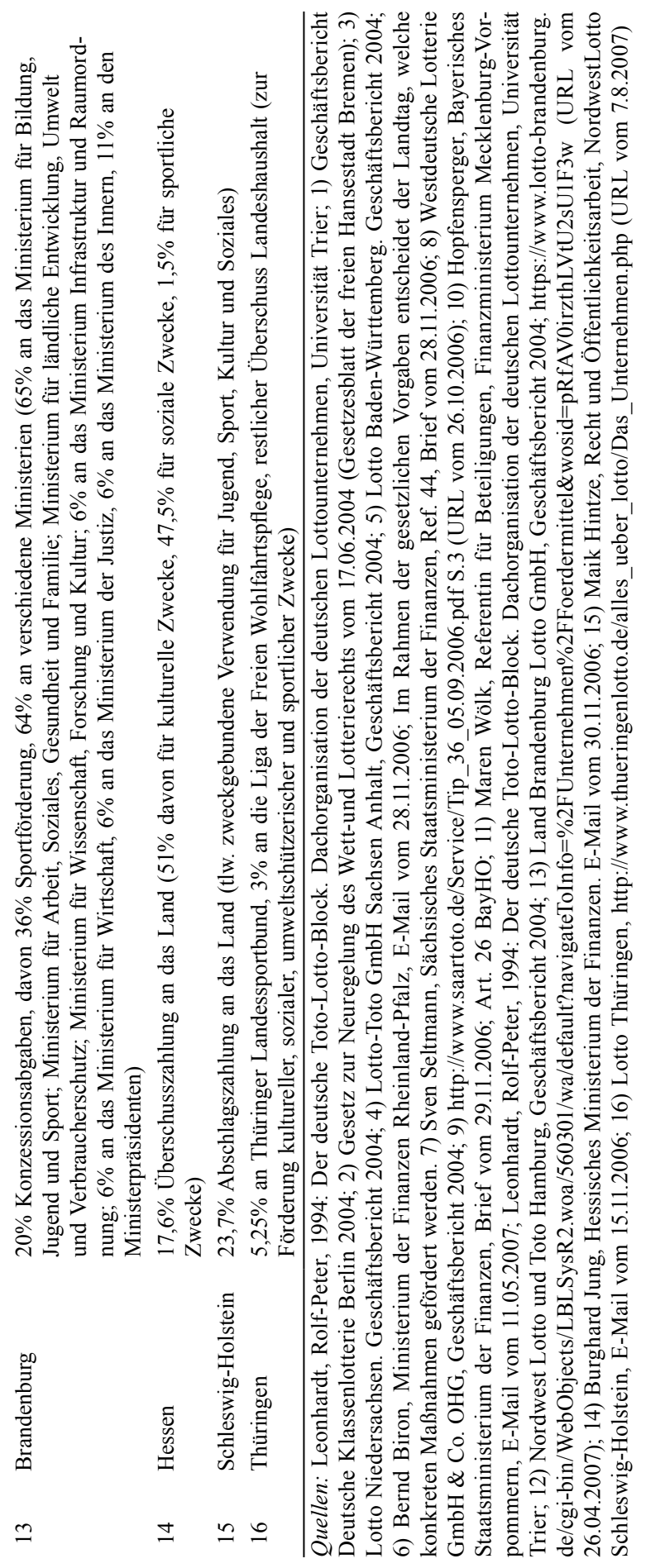


überein? Im Fragebogen haben wir neben der Teilnahme an der Lotterie innerhalb der letzten zwölf Monate auch die Teilnahme an Aktivitäten eines Sportvereins innerhalb dieses Zeitraumes abgefragt. Da der Grad der tatsächlichen Nutznießung der Sportförderung eine Größe ist, die sich jeder direkten Beobachtung entzieht, nehmen wir die Abfrage der Teilnahmeentscheidung als Indikator der individuellen Nutznießung der Lottomittel zur Förderung des Breitensports. ${ }^{19}$ Um nun die soziale Struktur der Nutznießer der Sportförderung mit der der Lottospieler vergleichen zu können, schätzen wir mit zwei Logitmodellen die individuelle Wahrscheinlichkeit der Zugehörigkeit beider Gruppen in Abhängigkeit diverser soziodemografischer Strukturmerkmale. Dazu verwenden wir die gleichen Merkmale, die bereits die Basis der ersten Analyse bildeten (vgl. Tabelle 1). Aus den modelltheoretisch geschätzten Wahrscheinlichkeiten erhalten wir nun auf Basis der Umfragedaten eine sozialstrukturell kanalisierte Wahrscheinlichkeitsverteilung der Mitgliedschaft beider Gruppen. Wie die in Abbildung 4 dargestellte gemeinsame Verteilung dieser Wahrscheinlichkeiten illustriert, stehen die geschätzten Zugehörigkeiten in einer deutlich negativen Korrespondenz zueinander (Pearson Korr. $=-0,410 ; \mathrm{p}<0,000)$. Das Maximum der Anpassungskurve einer in dieses Diagramm gelegten quadratischen Spezifikation zeigt etwa, dass die höchste mittlere Teilnahmewahrscheinlichkeit am Lottospiel eine weit unterdurchschnittliche mittlere Benefizwahrscheinlichkeit von etwa 0,25 impliziert, was einer Abweichung über 1,4 Standardabweichungen vom Mittelwert entspricht.

Lottospieler stimmen demnach sozialstrukturell nur sehr bedingt mit der Gruppe überein, die über die Sportförderung von den Lottomitteln profitieren. Je höher also die sozialstrukturell bedingte Wahrscheinlichkeit, Nutznießer der Sportförderung zu sein, desto geringer ist gleichzeitig die Wahrscheinlichkeit, Beitragszahler dieser Mittel zu sein. Die Ergebnisse dieser Analyse indizieren somit für den exemplarischen Fall der Breitensportförderung deutliche Belege für die Bestätigung der zweiten Hypothese.

In Ergänzung dieser Untersuchung interessiert uns nun im Folgenden eine Schätzung des monetären Nutzens aus der Sportförderung. Dieser ermittelt sich für jede Befragungsperson im Datensatz unter Verwendung der Vorgehensweise bei Borg und Mason (1988: 79 f.) und Rubenstein und Scafidi (2002: 233 f.) aus dem mit der modelltheoretisch vorhergesagten Wahrscheinlichkeit aktiver Teilnahme im Sportverein gewichteten Pro-Kopf-Aufkommen aus der Sportförderung. Daraus ergibt sich ein monetärer Schät-

19 Die Förderung von Sportvereinen durch Lottomittel richtet sich primär an den Breitensport. Durch unsere Frageformulierung ist es durchaus denkbar, dass wir eine geringere Anzahl von Personen erfassen, die zwar in Sportvereinen aktiv sind, zugleich aber nicht unmittelbar von der Förderung profitieren. Dies beträfe etwa Mitglieder in Tennis-, Golf- oder Schachclubs. Auch ist nicht auszuschließen, dass sich Personen irrtümlich als aktives Mitglied eines Sportvereins bezeichnen, faktisch aber privaten, kommerziell ausgerichteten Sportgesellschaften wie Fitness- oder Wellnessclubs angehören, die von der öffentlichen Sportförderung ausgeschlossen sind. Wir erfassen hier also möglicherweise eine bestimmte Anzahl an Personen, die keine Nutznießer der Sportförderung sind. Auf der anderen Seite erfassen wir nur die aktiven, nicht aber die passiven oder früheren Mitglieder in Sportvereinen. Demnach schließen wir ebenso einen geringen Prozentsatz von Nutznießern der Sportförderung mit diesem Instrument aus. Unser Instrument zur Erfassung der Nutznießung von Lottomitteln kann also nur als eine Annäherung verstanden werden, bei der wir ein gewisses, u. U. systematisch verlaufendes Messfehlerniveau nicht ausschließen können. 
Abbildung 4: Gemeinsame Verteilung der Beitrags- und Benefizwahrscheinlichkeiten

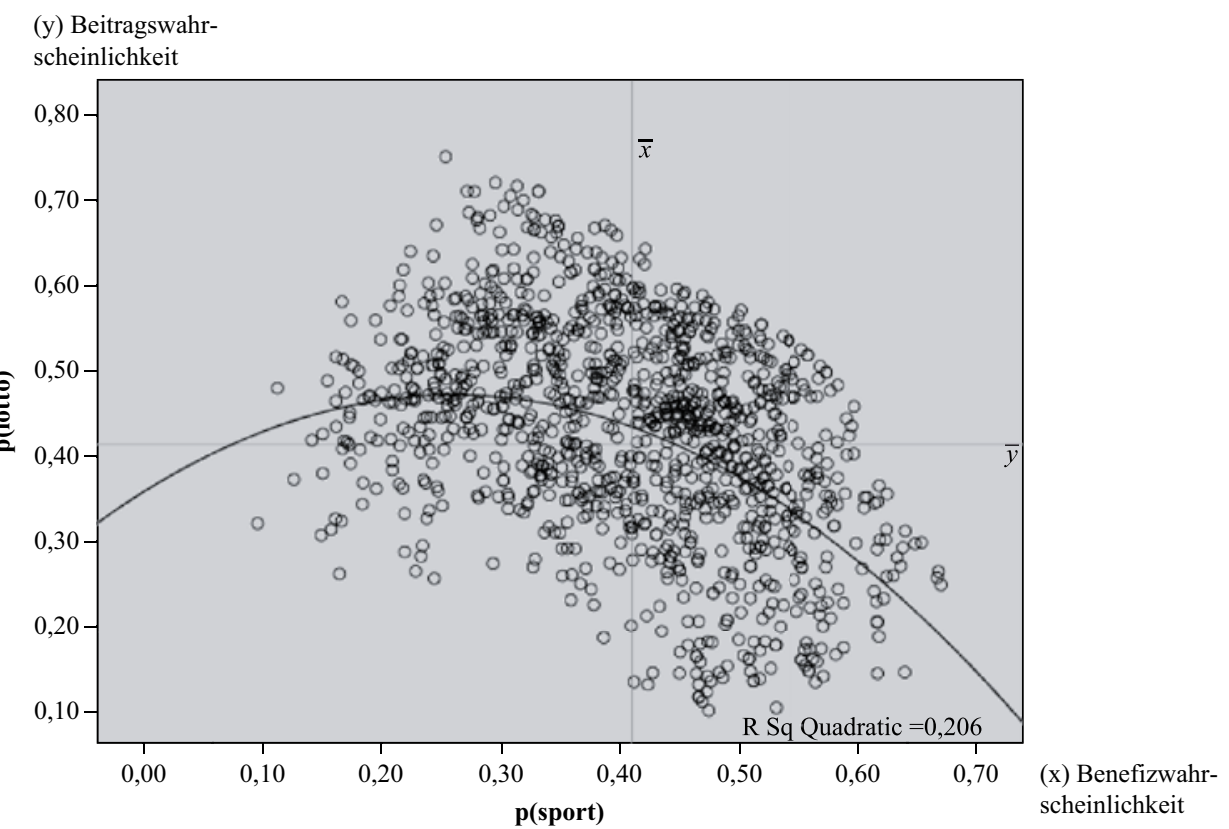

zer des individuellen Bruttonutzens aus der Sportförderung. Zugleich resultiert aus dem durchschnittlichen Anteil der Mittelverwendung zur Förderung des Breitensports, etwa 3 Prozent des Gesamtspieleinsatzes, am individuell verausgabten Spieleinsatz ein monetärer Schätzer für den pro Befragungsperson erbrachten Beitrag, der der Sportförderung zugute kommt. Die Differenz beider Werte über alle Fälle führt nun in eine Verteilung, die für jede Befragungsperson einen um den geleisteten Beitrag bereinigten monetären Schätzer des individuell erlangten Nettonutzens aus der Sportförderung anzeigt. Wie Abbildung 5 dokumentiert, erzielen Nichtspieler mit etwa 2,5 Euro einen positiven mittleren Monetärnutzen. Für Lottospieler hingegen liegt der Wert bei mittleren -4 Euro im Jahr, was bedeutet, dass Lottospieler im Schnitt mehr zahlen als sie zurückbekommen (s. unter Zeile „Total“). Es sind die Nichtspieler, die von der Verwendung der staatlichen Lotterieeinnahmen für den Breitensport profitieren.

Abbildung 5 zeigt weiter die Verteilung des mittleren Nettonutzens innerhalb verschiedener Kategorien sozialstruktureller Merkmale (Alter, Bildung und Einkommen). So erhalten insbesondere ältere Spieler, solche mit geringer Schulbildung und diejenigen im höchsten Einkommensquintil einen besonders ausgeprägten negativen Monetärnutzen. Letzterer Befund erklärt sich aus den höheren absoluten Ausgaben für das Lottospiel von Personen aus dem obersten Einkommensquintil. Bei den Nichtspielern sind die positiven Nutzenunterschiede zwischen den Kategorien der einzelnen soziodemographischen Gruppen nicht so stark ausgeprägt. Feststellen lässt sich jedoch ein ausgeprägter Nutzen bei den jüngeren Alterskohorten, die Sportvereine stärker nutzen.

Ein mit den Merkmalen Geschlecht, Bildung, Alter, Staatsangehörigkeit und Einkommen spezifiziertes Regressionsmodell zeigt auf, dass das Einkommen unter 
Abbildung 5: Geschätzter mittlerer Monetärnutzen aus der Breitensportförderung, in Euro/Jahr; nach soziodemografischen Gruppen; je für Lottospieler u. Nichtspieler

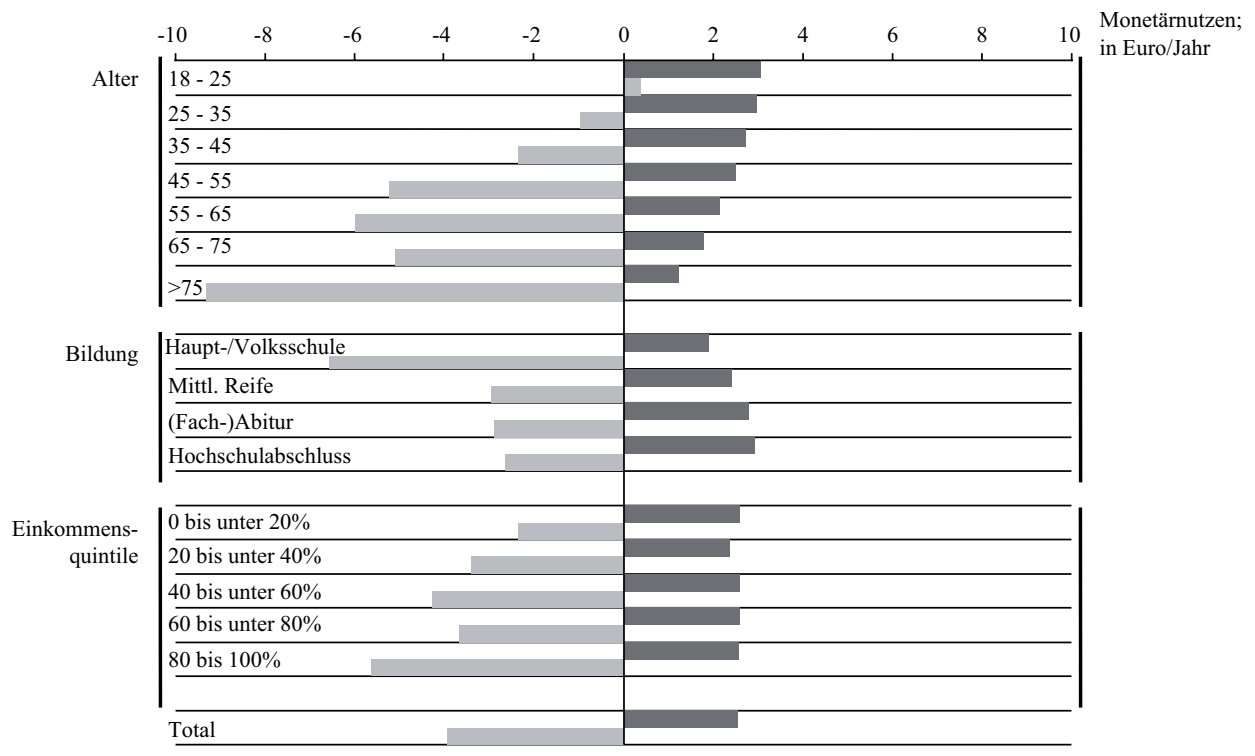

Konstanthaltung der übrigen Merkmale signifikant negativ mit dem geschätzten individuellen Nettobenefiz aus der Sportförderung variiert (vgl. Tabelle 4). Signifikant variiert weiter die Entscheidung zur Spielteilnahme: So bekommen Lottospieler im Schnitt etwa 6 Euro weniger aus der Sportförderung zurück als Nichtspieler (vgl. Modell 1 in Tabelle 4), während regelmäßige Spieler gar eine Negativbilanz von mehr als 9 Euro aufweisen (vgl. Modell 2). Als weitere Einflussmerkmale erweisen sich das Bildungsniveau, das Lebensalter sowie mit geringerer statistischer Deutlichkeit die Staatszugehörigkeit und das Geschlecht. So profitieren von der Sportförderung in stärkerem Maße jüngere, eher weibliche und vor allem höher gebildete Personen deutscher Staatsangehörigkeit, wohingegen Beitragszahler mit negativer Bilanz tendenziell männlich mit ethnischer Herkunft sind und vornehmlich aus unteren Bildungsschichten und älteren Geburtsjahrgängen stammen.

Bilanzierend lässt sich festhalten, dass die aus den Lotterieeinnahmen generierten Gelder über die Zweckbindung, wie hier am Fallbeispiel des Breitensports dargelegt, systematisch ungleich innerhalb sozialstrukturell relevanter Größen wie Einkommens- und vor allem auch Bildungsschichten umverteilt werden. Die im ersten Abschnitt dargelegten regressiven steuerlichen Effekte werden demnach durch die zweckgebundene Verwendung der Gelder nicht amortisiert oder abgefedert, sondern verstärken sich weiter. Dies würde ebenso gelten, so unsere Vermutung, hätten wir sämtliche zweckgebundene Verwendungsbereiche von Wohlfahrt über Kunst und Kultur in vergleichbarer Weise untersucht. Denn wahrscheinlich generiert zwar die nach der Sportförderung monetär 
Tabelle 4: Regressionsmodelle zur Schätzung des monetären Nettonutzens aus der Sportförderung

\begin{tabular}{|c|c|c|}
\hline & $\begin{array}{c}\text { (1) } \\
\text { Nettonutzen } \\
\text { (in Euro/Jahr) }\end{array}$ & $\begin{array}{c}\text { (2) } \\
\text { Nettonutzen } \\
\text { (in Euro/Jahr) }\end{array}$ \\
\hline Einkommen & $-0,283$ & $-0,182$ \\
\hline$(1=$ gering; $5=$ hoch $)$ & $(2,41)^{* * *}$ & $(1,74)^{*}$ \\
\hline Geschlecht & $-0,520$ & $-0,327$ \\
\hline$(1$ = männlich $)$ & $(\mathbf{1}, \mathbf{8 4})^{*}$ & $(1,28)$ \\
\hline Alter & $-0,064$ & $-0,036$ \\
\hline (in Jahren) & $(8,18)^{* * *}$ & $(5,36)^{* * *}$ \\
\hline Bildung & 0,693 & $\mathbf{0 , 5 5 4}$ \\
\hline$(1=$ gering; $5=$ hoch $)$ & $(4,61)^{* * * *}$ & $(4,09)^{* * * *}$ \\
\hline Staatsangehörigkeit & 2,613 & 2,620 \\
\hline$(1=$ deutsch $; 0=$ sonst $)$ & $(1,75)^{*}$ & $(1,94)^{*}$ \\
\hline Spielteilnahme & $-6,015$ & \\
\hline$(1=$ Lottospieler; $0=$ Nichtspieler $)$ & $(18,48)^{* * *}$ & \\
\hline Spielhäufigkeit 1 & & $-9,276$ \\
\hline$(1=$ regelmäßig; $0=$ sonst $)$ & & $(17,13)^{* * *}$ \\
\hline Spielhäufigkeit 2 & & $-2,137$ \\
\hline$(1=$ unregelmäßig; $0=$ sonst $)$ & & $(18,25)^{* * * *}$ \\
\hline \multirow{2}{*}{\multicolumn{3}{|c|}{$\begin{array}{l}\text { Spielhäufigkeit } 3 \\
\text { (Nichtspieler = Referenzkategorie) }\end{array}$}} \\
\hline & & \\
\hline \multirow[t]{2}{*}{ Konstante } & 2,047 & 0,804 \\
\hline & $(1,38)$ & $(0,59)$ \\
\hline $\mathrm{N}$ & 1094 & 1094 \\
\hline Adj. R-Quadrat & 0,29 & 0,39 \\
\hline
\end{tabular}

Signifikanz: ${ }^{*} \mathrm{p}<0,1 ;{ }^{* *} \mathrm{p}<0,05 ;{ }^{* * *} \mathrm{p}<0,01$ (zweiseitiger Test); in Klammern: $\mathrm{z}^{-}$Statistiken.

wichtigste Mittelverwendung für Wohlfahrtsorganisationen mildernde Umverteilungswirkungen, doch ist von der übrigen Vergabestruktur in Bereichen wie Kunst und Kultur (Museen, Denkmalförderung, etc.) eher mit stärkeren Effekten zu rechnen, als sie hier für das Beispiel des Breitensports festgestellt wurden. Das von uns exemplarisch gewählte Beispiel dürfte, was die sozialstrukturelle Partizipation anbelangt, wohl in der Mitte der sonst vorkommenden Verwendungsbereiche liegen.

\section{Schluss}

Wir konnten in diesem Artikel auf der Grundlage der von uns erhobenen Daten zeigen, dass das staatliche Lotteriespiel eine Form regressiver Besteuerung darstellt und damit das in der Steuertheorie als normatives Kriterium zur Beurteilung einer Steuer angelegte Leistungsfähigkeitsprinzip verletzt. Lotteriespieler mit geringerem Einkommen tragen in signifikant höherem Maß zum Steueraufkommen bei. Dieser Befund bestätigt die Ergebnisse internationaler Studien zu dem Thema, die vornehmlich in den angelsächsi- 
schen Ländern durchgeführt wurden. Darüber hinaus können wir Zusammenhänge zum Bildungsniveau der Spieler, der Beschäftigung, dem Lebensalter und dem Migrationshintergrund aufzeigen.

Im zweiten Teil der empirischen Untersuchung haben wir die Übereinstimmung der als Konzessionsabgaben zweckgebunden speziellen Verwendungen zugeführten staatlichen Einnahmen aus dem Lotto mit dem tatsächlichen Nachfragerverhalten der Spieler in Beziehung gesetzt. Am Beispiel der Förderung des Breitensports, der die höchsten Zuwendungen aus den Lotterieeinnahmen erhält, konnten wir zeigen, dass die Lotteriespieler die finanzierten Angebote weit unterdurchschnittlich nutzen. Pointiert zusammengefasst bezahlen die Lottospieler die Kosten für Angebote, die von den Nichtspielern genutzt werden. Damit verletzt die Verwendungsseite der zweckgebundenen Lotterieeinnahmen auch das Äquivalenzprinzip als zweites normatives Gütekriterium für eine Steuer und verstärkt den regressiven Effekt des Lottospiels. Wenngleich letztendlich die Gesamtwirkung des Steuersystems für dessen normative Bewertung ausschlaggebend ist, erscheinen die Lotterieeinnahmen des Staates doch problematisch, da in Debatten um die Steuerprogression solche regressiven Teile des Systems unbeachtet bleiben.

Welche Schlussfolgerungen für die Gestaltung des Lotteriespiels lassen sich aus diesen Ergebnissen ziehen? In der Literatur zu dem Thema werden in starkem Maß Vorschläge unterbreitet, wonach die regressiven Besteuerungseffekte durch eine Ausweitung des Spiels kompensiert werden sollen. So sollen u. a. Lotterieprodukte stärker beworben und dadurch breitere Bevölkerungsschichten erreicht werden (vgl. Borg/Stranahan 2005). ${ }^{20}$ Angesichts der politischen Diskussion um die Bekämpfung von Spielsucht erscheint eine Ausweitung der Nachfragestruktur jedoch undenkbar. Die Bewerbung staatlicher Glücksspielprodukte ist mit dem gesetzlichen Kanalisierungsauftrag nur dann vereinbar, wenn diese dazu dient, illegale Glücksspielaktivitäten zu unterwandern und einzudämmen (vgl. Hecker 2004). Eine Alternative zielt auf die Ausgabenseite der durch das Lotteriespiel staatlich vereinnahmten Gelder. Wenn es sich so verhält, dass das Steueraufkommen überproportional von Beziehern unterer Einkommen aufgebracht wird, dann sollte es Verwendungen zugeführt werden, von denen genau diese Personengruppe auch überproportional profitiert. Ein erster Schritt zum Abbau der Regressivität auf der Verwendungsseite wäre die generelle Zuführung der staatlichen Lotterieeinnahmen in die Landeshaushalte, deren Verwendung den Präferenzen für verschiedene öffentliche Güter eher genügt. Ein darüber hinausgehender Schritt wäre die Verausgabung als zweckgebundene Mittel im Bereich der nichtgymnasialen schulischen und vorschulischen Bildung, wovon gerade einkommensschwächere Schichten profitieren würden.

Eine Auflösung des staatlichen Monopols des Zahlenlottos und eine Liberalisierung des Marktes schließlich, die zurzeit politisch und juristisch diskutiert werden, ließe keine Reduzierung der regressiven Effekte des Lottospiels erwarten. Zum einen würden lediglich private anstelle staatlicher Gewinne entstehen, die im obersten Einkommensquintil anfallen und somit eine direkte soziale Umverteilung bewirken würden. Zum

20 Weitere Vorschläge, die auf die schichtbezogene Ausweitung des Spiels zur Verminderung der Regressivität abzielen, ergeben sich bei Garvía (2007), Garvía/Guillén (2007), Miyazaki et al. (1998) und Oster (2004). 
anderen müsste aufgrund des internationalen Wettbewerbs zwischen den Lotterien die Besteuerung in Deutschland durchgeführter Lotterien vermutlich reduziert werden, so dass weniger Geld für eine Verwendung nach dem Äquivalenzprinzip überhaupt zur Verfügung stünde.

\section{Literatur}

Albers, Norman, 1993: Ökonomie des Glücksspielmarktes in der Bundesrepublik Deutschland. Berlin: Duncker \& Humblot.

Beckert, Jens/Lutter, Mark, 2007: Wer spielt, hat schon verloren? Zur Erklärung des Nachfrageverhaltens auf dem Lottomarkt, in: Kölner Zeitschrift für Soziologie und Sozialpsychologie 59, 240-270.

Blalock, Garrick/Just, David R./Simon, Daniel H., 2007: Hitting the Jackpot or Hitting the Skids: Entertainment, Poverty, and the Demand for State Lotteries, in: American Journal of Economics and Sociology 66, 545-570.

Bloch, Herbert A., 1951: The Sociology of Gambling, in: The American Journal of Sociology 57, 215-221.

Borg, Mary O./Mason, Paul M., 1988: The Budgetary Incidence of a Lottery to Support Education, in: National Tax Journal 41, 75-85.

Borg, Mary O./Mason, Paul M./Shapiro, Stephen L., 1991: The Economic Consequences of State Lotteries. New York: Praeger.

Borg, Mary O./Stranahan, Harriet A., 2005: Does Lottery Advertising Exploit Disadvantaged and Vulnerable Markets?, in: Business Ethics Quarterly 15, 23-35.

Braidfoot, Larry, 1985: Gambling. A Deadly Game. Nashville, Tennessee: Broadman Press.

Brinner, Roger E./Clotfelter, Charles T., 1975: An Economic Appraisal of State Lotteries, in: National Tax Journal 28, 395-404.

Brown, Daniel J./Kaldenberg, Dennis O./Browne, Beverly A., 1992: Socioeconomic-Status and Playing the Lotteries, in: Sociology and Social Research 76, 161-167.

Brümmerhoff, Dieter, 1992: Finanzwissenschaft. München/Wien: Oldenbourg.

Bundesministerium der Finanzen, 2006: Kassenmäßige Steuereinnahmen nach Steuerarten. http:// www.bundesfinanzministerium.de/cln 06/nn 4158/DE/Steuern/Steuerschaetzung einnahmen/Steuereinnahmen/0601011a6002.html (URL vom 16.01.2007).

Calmus, Thomas W., 1981: Measuring the Regressivity of Gambling Taxes, in: National Tax Journal 34, 267-270.

Campbell, Colin, 1987: The Romantic Ethic and the Spirit of Modern Consumerism. Oxford, UK: B. Blackwell.

Clotfelter, Charles T., 1979: Regressivity of State-Operated Numbers Games, in: National Tax Journal 32, 543-548.

Clotfelter, Charles T., 2000. Do Lotteries Hurt the Poor? Well, Yes and No. A Summary of Testimony Given to the House Select Committee on a State Lottery.

Clotfelter, Charles T./Cook, Philip J., 1987: Implicit Taxation in Lottery Finance, in: National Tax Journal 40, 533-546.

Clotfelter, Charles T./Cook, Philip J., 1991: Selling Hope. State Lotteries in America. Cambridge, MA: Harvard University Press.

Cohen, Lloyd R., 2001: The Lure of the Lottery, in: Wake Forest Law Review 36, 705-745.

Cosgrave, Jim/Klassen, Thomas R., 2001: Gambling against the State: The State and the Legitimation of Gambling, in: Current Sociology 49, 1-15.

Deutsche Hauptstelle für Suchtfragen e.V. (Hrsg.), 2006: Jahrbuch Sucht 2006. Geesthacht: Neuland. 
Deutscher Sportbund, 2005. Bestandserhebung 2005. Frankfurt a. M.

Devereux, Edward C. Jr., 1980 (1949): Gambling and the Social Structure. A Sociological Study of Lotteries and Horse Racing in Contemporary America. New York: Arno Press.

Downes, D. M./Davies, B. P./David, M. E./Stone, P., 1976: Gambling, Work and Leisure. London: Routledge and Kegan Paul Ltd.

Engelhardt, Henriette, 1999: Lineare Regression mit Selektion: Möglichkeiten und Grenzen der Heckman-Korrektur, in: Kölner Zeitschrift für Soziologie und Sozialpsychologie 51, 706-723.

Faulbaum, Frank/Deutschmann, Marc/Kleudgen, Martin, 2003: Computerunterstütztes Pretesting von CATI-Fragebögen: Das CAPTIQ-Verfahren, in: ZUMA-Nachrichten 52, 20-34.

Frey, James H., 1984: Gambling: A Sociological Review, in: Annals of the American Academy of Political and Social Science 474, 107-121.

Friedman, Milton/Savage, L. J., 1948: The Utility Analysis of Choices Involving Risk, in: The Journal of Political Economy 56, 279-304.

Gabler, Siegried/Häder, Sabine, 1997: Überlegungen zu einem Stichprobendesign für Telefonumfragen in Deutschland, in: ZUMA-Nachrichten 41, 7-18.

Garvia, Roberto, 2007: Syndication, Institutionalization, and Lottery Play, in: American Journal of Sociology 113, 603-652.

Garvia, Roberto/Guillén, Mauro F., 2007: Socioeconomic Determinants of Syndicate Play (Manuskript).

Groves, Robert M./Dillman, Don A./Eltinge, John L./Little, Roderick J.A., 2002: Survey Nonresponse. New York: John Wiley and Sons, INC.

Gujarati, Damodar N., 2003: Basic Econometrics. Boston: McGraw Hill.

Haller, Heinz, 1981: Die Steuern. Tübingen: Mohr.

Hansen, Ann, 1995: The Tax Incidence of the Colorado State Lottery Instant Game, in: Public Finance Quarterly 23, 385-398.

Hansen, Ann/Miyazaki, Anthony D./Sprott, David E., 2000: The Tax Incidence of Lotteries: Evidence from Five States, in: Journal of Consumer Affairs 34, 182-203.

Heavey, Jerome F., 1978: The Incidence of State Lottery Taxes, in: Public Finance Quarterly 6, 415-426.

Hecker, Manfred, 2004: Das staatliche Glücksspiel zwischen ordnungspolitischer Aufgabenstellung und effizienzorientiertem Marketing: Widerspruch in sich oder Königsweg?, in: Westlotto (Hrsg.), Geschäftsbericht 2004. Münster: Westdeutsche Lotterie GmbH \& Co. OHG, 4-9.

Heckman, James J., 1979: Sample Selection Bias as a Specification Error, in: Econometrica 47, 153-161.

Husz, Orsi, 2002: Private Dreams and Public Expectations. Lotteries and Dilemmas of Progress and Social Welfare in Early 20th-Century Sweden, in: Journal of Consumer Culture 2, 53-79.

Jackson, Raymond, 1994: Demand for Lottery Products in Massachusetts, in: Journal of Consumer Affairs 28, 313-325.

Kahneman, Daniel/Tversky, Amos, 1979: Prospect Theory - Analysis of Decision under Risk, in: Econometrica 47, 263-291.

Karcher, Alan J., 1989: Lotteries. New Brunswick: Transaction Publishers.

Karcher, Alan J., 1992: State Lotteries, in: Society 29, 51-56.

King, Kim M., 1985: Gambling: Three Forms and Three Explanations, in: Sociological Focus $18,235-248$.

Kitchen, Harry/Powells, Scott, 1991: Lottery Expenditures in Canada: A Regional Analysis of Determinants and Incidence, in: Applied Economics Letters 23, 1845-1852.

Klein, Thomas, 2005: Sozialstrukturanalyse. Reinbek bei Hamburg: Rowohlt. 
Koza, John R., 1982: The Myth of the Poor Buying Lottery Tickets, in: Public Gaming 10, 31-50.

Leonhardt, Rolf-Peter, 1994. Der deutsche Toto-Lotto-Block. Dachorganisation der deutschen Lottounternehmen. Arbeitspapier Nr. 21 des Schwerpunktes Finanzwissenschaft/Betriebswirtschaftliche Steuerlehre, Universität Trier.

Liebig, Stefan/Mau, Steffen, 2005: Wann ist ein Steuersystem gerecht? Einstellungen zu allgemeinen Prinzipien der Besteuerung und zur Gerechtigkeit der eigenen Steuerlast, in: Zeitschrift für Soziologie 34, 468-491.

Livernois, J. R., 1987: The Redistributive Effects of Lotteries - Evidence From Canada, in: Public Finance Quarterly 15, 339-351.

Long, J. Scott, 1997: Regression Models for Categorical and Limited Dependent Variables. Thousand Oaks: Sage Publications.

McCaffery, Edward J., 1994: Why People Play Lotteries and Why It Matters, in: Wisconsin Law Review 71, 71-122.

McConkey, C. William/Warren, William E., 1987: Psychographic and Demographic Profiles of State Lottery Ticket Purchasers, in: Journal of Consumer Affairs 21, 314-327.

Meyer, Gerhard, 2006: Glücksspiel - Zahlen und Fakten, in: Deutsche Hauptstelle für Suchtfragen e.V. (Hrsg.), Jahrbuch Sucht 2006. Geesthacht: Neuland, 114-128.

Meyer, Gerhard/Bachmann, Meinolf, 2005: Spielsucht. Ursachen und Therapie. Heidelberg: Springer.

Mikesell, John L., 1989: A Note on the Changing Incidence of State Lottery Finance, in: Social Science Quarterly 70, 513-521.

Miyazaki, Anthony D./Hansen, Ann/Sprott, David E., 1998: A Longitudinal Analysis of Incomebased Tax Regressivity of State-sponsored Lotteries, in: Journal of Public Policy \& Marketing 17, 161-172.

Nibert, David, 2000: Hitting the Jackpot. New York: Monthly Review Press.

Nibert, David, 2006: State Lotteries and the Legitimation of Inequality, in: Cosgrave, James F. (Hrsg.), The Sociology of Risk and Gambling Reader. New York: Routledge, 319-338.

Oster, Emily, 2004: Are All Lotteries Regressive? Evidence from the Powerball, in: National Tax Journal 57, 179-187.

Paul, Wolfgang, 1978: Erspieltes Glück: Deutsche Klassenlotterie Berlin.

Price, Donald I./Novak, E. Shawn, 1999: The Tax Incidence of Three Texas Lottery Games: Regressivity, Race, and Education, in: National Tax Journal 52, 741-751.

Reith, Gerda, 1999: The Age of Chance. Gambling in Western Culture. London: Routledge.

Rogers, Paul, 1998: The Cognitive Psychology of Lottery Gamblings. A Theoretical Review, in: Journal of Gambling Studies 14, 111-134.

Rogers, Paul/Webley, Paul, 2001: „It could be us!“”: Cognitive and Social Psychological Factors in UK National Lottery Play, in: Applied Psychology 50, 181-199.

Rubenstein, Ross/Scafidi, Benjamin, 2002: Who Pays and Who Benefits? Examining the Distributional Consequences of the Georgia Lottery for Education, in: National Tax Journal 55, 223-238.

Sawkins, John W./Dickie, Valerie A., 2002: National Lottery Participation and Expenditure: Preliminary Results Using a Two Stage Modelling Approach, in: Applied Economics Letters 9, 769-773.

Scott, Frank/Garen, John, 1994: Probability of Purchase, Amount of Purchase, and the Demographic Incidence of the Lottery Tax, in: Journal of Public Economics 54, 121-143.

Smith, Ronald W./Preston, Frederick W./Humphries, Harry L., 1976: Alienation from Work. A Study of Casino Card Dealers, in: Eadington, William R. (Hrsg.), Gambling and Society. Springfield, Ill.: Thomas, 229-246.

Spindler, Charles J., 1995: The Lottery and Education: Robbing Peter to Pay Paul?, in: Public Budgeting and Finance 15, 54-62. 
Spiro, Michael H., 1974: Tax Incidence of Pennsylvania Lottery, in: National Tax Journal 27, 57-61.

Staatliche Toto-Lotto GmbH Baden Württemberg (Hrsg.), 1998: 50 Jahre Toto-Lotto Baden Württemberg. Stuttgart: Kohlhammer.

Statistisches Bundesamt, 2004. Demographische Standards. Wiesbaden.

Stranahan, Harriet A./Borg, Mary O., 1998: Separating the Decisions of Lottery Expenditures and Participation: A Truncated Tobit Approach, in: Public Finance Review 26, 99-117.

Stranahan, Harriet A./Borg, Mary O., 2004: Some Futures are Brighter than Others: The Net Benefits Received by Florida Bright Futures Scholarship Recipients, in: Public Finance Review 32, 105-126.

Suits, Daniel B., 1977a: Gambling Taxes: Regressivity and Revenue Potential, in: National Tax Journal 30, 19-35.

Suits, Daniel B., 1977b: Measurement of Tax Progressivity, in: American Economic Review 67, 747-752.

Tec, Nechama, 1964: Gambling in Sweden. Totowa: Bedminster Press.

Tettinger, Peter J./Ennuschat, Jörg, 1999: Grundstrukturen des deutschen Lotterierechts. München: Vahlen.

Tobin, James, 1958: Estimation of Relationships for Limited Dependent Variables, in: Econometrica 26, 24-36.

Tolkemitt, Till, 2002: Die deutsche Glücksspielindustrie. Eine wirtschaftswissenschaftliche Analyse mit rechtspolitischen Schlussfolgerungen. Frankfurt a. M.: Lang.

Vaillancourt, Francois/Grignon, Julie, 1988: Canadian Lotteries as Taxes: Revenues and Incidence, in: Canadian Tax Journal (Revue Fiscale Canadienne) 36, 369-388.

Vasche, Jon D., 1985: Are Taxes on Lotteries Too High, in: Journal of Policy Analysis and Management 4, 269-271.

Beckert, Jens, 1967, Prof. Dr., Direktor am Max-Planck-Institut für Gesellschaftsforschung in Köln. Forschungsgebiete: Wirtschafts- und Organisationssoziologie, Soziologie der Erbschaft, soziologische Theorie. Veröffentlichungen: Inherited Wealth. Princeton University Press 2008; Märkte als soziale Strukturen. Frankfurt a. M. 2007 (Hrsg. mit Rainer Diaz-Bone und Heiner Ganßmann); The Longue Durée of Inheritance Law. Discourses and Institutional Development in France, Germany, and the United States since 1800, in: Archives Européennes de Sociologie XLVIII, 1, 79-120.

Lutter, Mark, 1976, Dipl. Soz.-Wiss., wissenschaftlicher Mitarbeiter am Max-Planck-Institut für Gesellschaftsforschung in Köln. Forschungsgebiete: Wirtschaftssoziologie, Sozialstrukturanalyse, Stichprobentheorie, statistische Modellierung. Veröffentlichungen: Wer spielt, hat schon verloren? Zur Erklärung des Nachfrageverhaltens auf dem Lottomarkt, in: Kölner Zeitschrift für Soziologie und Sozialpsychologie 59: 240-270 (mit Jens Beckert). 